\title{
Linear-Time Approximation for Maximum Weight Matching
}

\author{
RAN DUAN, Max-Planck-Institut für Informatik \\ SETH PETTIE, University of Michigan
}

\begin{abstract}
The maximum cardinality and maximum weight matching problems can be solved in $\tilde{O}(m \sqrt{n})$ time, a bound that has resisted improvement despite decades of research. (Here $m$ and $n$ are the number of edges and vertices.) In this article, we demonstrate that this " $m \sqrt{n}$ barrier" can be bypassed by approximation. For any $\epsilon>0$, we give an algorithm that computes a $(1-\epsilon)$-approximate maximum weight matching in $O\left(m \epsilon^{-1} \log \epsilon^{-1}\right)$ time, that is, optimal linear time for any fixed $\epsilon$. Our algorithm is dramatically simpler than the best exact maximum weight matching algorithms on general graphs and should be appealing in all applications that can tolerate a negligible relative error.
\end{abstract}

Categories and Subject Descriptors: G.2.2 [Discrete Mathematics]: Graph Theory—Graph algorithms

General Terms: Algorithms, Theory

Additional Key Words and Phrases: Matching, assignment, approximation

ACM Reference Format:

Duan, R. and Pettie, S. 2014. Linear-time approximation for maximum weight matching. J. ACM 61, 1, Article 1 (January 2014), 23 pages.

DOI: http://dx.doi.org/10.1145/2529989

\section{INTRODUCTION}

Graph matching is one of the most well-studied problems in combinatorial optimization. The original motivations of the problem were to minimize transportation costs [Hitchcock 1941; Kantorovitch 1942] and optimally assign personnel to job positions [Easterfield 1946; Thorndike 1950]. Over the years, matching algorithms have found applications in scheduling, approximation algorithms, network switching, and as key subroutines in other optimization algorithms, for example, undirected shortest paths [Lawler 1976], planar max cut [Hadlock 1975; Orlova and Dorfman 1972], Chinese postman tours [Edmonds and Johnson 1973; Kwan 1962], and metric traveling salesman [Christofides 1976]. In some applications it is not critical that the algorithm produce an exactly optimum solution. In this article, we explore the extent to which this freedom-not demanding exact solutions-allows us to design simpler and more efficient algorithms. ${ }^{1}$

\footnotetext{
${ }^{1}$ Many of the articles cited in this article can be found on the second author's homepage: web.eecs.umich.edu/ pettie/matching/.
}

\begin{abstract}
This work was supported by NSF Career grant no. CCF-0746673, NSF grant no. CCF-1217338, and a grant from the US-Israel Binational Science Foundation. R. Duan was supported by an Alexander von Humboldt Postdoctoral Fellowship.

Authors' addresses: R. Duan, Max-Planck-Institut für Informatik, Stuhlsatzenhausweg 85, 66123 Saarbrücken, Germany; email: duanr02@gmail.com; S. Pettie, Department of Electrical Engineering and Computer Science, University of Michigan, 2260 Hayward Street, Ann Arbor, MI 48109; email: pettie@umich.edu.

Permission to make digital or hard copies of part or all of this work for personal or classroom use is granted without fee provided that copies are not made or distributed for profit or commercial advantage and that copies show this notice on the first page or initial screen of a display along with the full citation. Copyrights for components of this work owned by others than ACM must be honored. Abstracting with credit is permitted. To copy otherwise, to republish, to post on servers, to redistribute to lists, or to use any component of this work in other works requires prior specific permission and/or a fee. Permissions may be requested from Publications Dept., ACM, Inc., 2 Penn Plaza, Suite 701, New York, NY 10121-0701 USA, fax +1 (212) 869-0481, or permissions@acm.org.
\end{abstract}

(C) 2014 ACM 0004-5411/2014/01-ART1 $\$ 15.00$

DOI : http://dx.doi.org/10.1145/2529989 
In order to discuss prior work with precision we must introduce some notation and terminology. The input is a weighted graph $G=(V, E, w)$ where $n=|V|$ and $m=|E|$ are the number of vertices and edges and $w$ is the edge weight function. If $w$ assigns integer (rather than real) weights, let $N$ be the largest magnitude of a weight. An unweighted graph is one for which $w(e)=1$ for all $e \in E$. A matching is a set of vertex-disjoint edges and a perfect matching is one in which all vertices are matched. The weight of a matching is the sum of its edge weights. We use MWM (and MWPM) to denote the problem of finding a maximum weight (perfect) matching, as well as the matching itself. We use MCM for the cardinality (unweighted) version of the problem. The MWPM problem on bipartite graphs is often called the assignment problem.

The MWPM and MWM problems are reducible to each other. Given an instance $G$ of MWM, let $G^{\prime}$ consist of two copies of $G$ with zero-weight edges connecting copies of the same vertex. Clearly a MWPM in $G^{\prime}$ corresponds to a pair of MWMs in $G$. In the reverse direction, if $G$ is an instance of MWPM with weight function $w$, one can find the MWM of $G$ using the weight function $w^{\prime}(e)=w(e)+n N$. Maximum weight matchings with respect to $w^{\prime}$ necessarily have maximum cardinality. Call a matching $\delta$-approximate, where $\delta \in[0,1]$, if its weight is at least a factor $\delta$ of the optimum matching. Let $\delta$-MWM (and $\delta$-MCM) be the problem of finding $\delta$-approximate maximum weight (cardinality) matching, as well as the matching itself. It is important to note that the reductions between MWPM to MWM do not work for the approximate versions of these problems since the approximation may compromise perfection. In this article, we only consider approximations of the MWM problem. Consequently, our algorithms cannot be used in applications that call for perfect matchings such as Lawler [1976], Orlova and Dorfman [1972], Hadlock [1975], Edmonds and Johnson [1973], Kwan [1962], and Christofides [1976].

Tables I, II, and III give an at-a-glance history of exact matching algorithms. Algorithms are dated according to their initial publication, and are included either because they establish a new time bound, or employ a noteworthy technique, or are of historical interest. Table IV gives a history of approximate MCM and MWM algorithms.

\subsection{Algorithms for Bipartite Graphs}

The MWM problem is expressible as the following integer linear program, where $x$ represents the incidence vector of the matching.

$$
\begin{array}{llr}
\text { maximize } & \sum_{e \in E} w(e) x(e) & \\
\text { subject to } & 0 \leq x(e) \leq 1 & \forall e \in E \\
& \sum_{e=\left(u, u^{\prime}\right) \in E} x(e) \leq 1 & \forall u \in V \\
& x(e) \text { is an integer } & \\
& &
\end{array}
$$

It is well known that in bipartite graphs the integrality requirement (2) is redundant, that is, the basic feasible solutions of the LP (1) are nonetheless integral. See Birkhoff [1946] and Dantzig [1951]. The dual of (1) is

$$
\begin{array}{rlr}
\text { minimize } & \sum_{u \in V} y(u) & \\
\text { subject to } & y(e) \geq w(e) & \forall e \in E \\
& y(u) \geq 0 & \forall u \in V \\
\text { where, by definition, } & y(u, v) \stackrel{\text { def }}{=} y(u)+y(v) . &
\end{array}
$$


Table I. Cardinality Matching

\begin{tabular}{|c|c|c|}
\hline Year & Authors & Time Bound \& Notes \\
\hline & folklore/trivial & BIPARTITE \\
\hline 1965 & & $m n^{2}$ \\
\hline 1965 & Witzgall \& Zahn & \\
\hline 1969 & Balinski & \\
\hline 1976 & Gabow & $m n$ or $n^{3}$ \\
\hline 1976 & Lawler & IIn $01 \pi$ \\
\hline 1976 & Karzanov & \\
\hline 1971 & Hopcroft \& Karp & RIDA DTITE \\
\hline 1973 & Dinic \& Karzanov & BIPARTITE \\
\hline 1980 & Micali \& Vazirani & $m \sqrt{n}$ \\
\hline 1991 & Gabow \& Tarjan & $m v \pi$ \\
\hline 1981 & Ibarra \& Moran & $n^{\omega}$ CARDINALITY ONLY,RANDOMIZED,BIPARTITE \\
\hline 1989 & Rahin \& Vazirani & CARDINALITY ONLY,RANDOMIZED \\
\hline 1000 & Mavill e vazil ant & RANDOMIZED \\
\hline 1991 & Alt, Blum, Mehlhorn \& Paul & $n \sqrt{n m / \log n}$ \\
\hline 1991 & Feder \& Motwani & BIPABTITE $\kappa=\frac{\log n}{2}$ \\
\hline 1997 & Goldberg \& Kennedy & DIFAnIIL, $\kappa-\overline{\log \left(n^{2} / m\right)}$ \\
\hline 1996 & Cheriyan \& Mehlhorn & $n^{2}+n^{5 / 2} / w$ BIPARTITE, $w=$ machine word size \\
\hline 2004 & Goldberg \& Karzanov & $m \sqrt{n} / \kappa$ \\
\hline 2004 & Mucha \& Sankowski & RANDOMIZED \\
\hline 2006 & Harvey & \\
\hline
\end{tabular}

Note: Here $\omega<2.373$ is the exponent of $n \times n$ matrix multiplication [Williams 2012]. The $m n$ running time on general graphs depends on a special union-find data structure [Gabow and Tarjan 1985] developed later. Without it, the running time would be $m n \alpha(m, n)$, where $\alpha$ is the inverse-Ackermann function.

In the MWPM problem $\sum_{e=\left(u, u^{\prime}\right)} x(e)=1$ holds with equality in the primal and $y(u)$ is unconstrained in the dual. Kuhn's [1955a, 1956] publication of the Hungarian method stimulated research on this problem from an algorithmic perspective, but it was not without precedent. Kuhn noted that the algorithm was latent in the work of Hungarian mathematicians König and Egerváry. ${ }^{2}$ However, the history goes back even further. A recently rediscovered article of Jacobi from 1865 describes a variant of the Hungarian algorithm; see Ollivier [2009]. Although Kuhn's algorithm self-evidently runs in polynomial time, this mark of efficiency was noted later: Munkres [1957] showed that $O\left(n^{4}\right)$ time is sufficient. Independent of Kuhn's work, Gleyzal [1955] discovered a polynomialtime cycle-canceling algorithm for the assignment problem and von Neumann [1953] gave a reduction from the assignment problem to finding the optimum strategy in a zero-sum bimatrix game, which can be solved in polynomial time [Brown and von Neumann 1950].

The search for faster assignment algorithms began in earnest in the 1960s. Dinic and Kronrod [1969] gave an $O\left(n^{3}\right)$-time algorithm and Edmonds and Karp [1972] and Tomizawa [1971] observed that assignment is reducible to $n$ single-source shortest

\footnotetext{
${ }^{2}$ A translation of Egerváry's work appears in Kuhn [1955b].
} 
Table II. Weighted Matching: Bipartite Graphs

\begin{tabular}{|c|c|c|}
\hline Year & Authors & Time Bound \& Notes \\
\hline 1946 & Easterfield & $2^{n} \operatorname{poly}(n)$ \\
\hline 1953 & von Neumann & \\
\hline 1955 & Kuhn & \\
\hline 1955 & Gleyzal & $\operatorname{poly}(n)$ \\
\hline 1957 & Munkres & \\
\hline 1964 & Balinski \& Gomory & \\
\hline 1969 & Dinic \& Kronrod & $n^{3}$ \\
\hline $\begin{array}{l}1970 \\
1971\end{array}$ & $\begin{array}{l}\text { Edmonds \& Karp } \\
\text { Tomizawa }\end{array}$ & 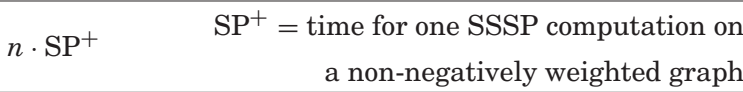 \\
\hline 1975 & Johnson & $d=2+m / n$ \\
\hline 1983 & Gabow & $\begin{array}{lr}m n^{3 / 4} \log N & \text { INTEGER WEIGHTS } \\
N m \sqrt{n} & \text { MWM ONLY, INTEGER WEIGHTS }\end{array}$ \\
\hline 1984 & Fredman \& Tarjan & $m n+n^{2} \log n$ \\
\hline $\begin{array}{l}1988 \\
1992 \\
1997 \\
2012\end{array}$ & $\begin{array}{l}\text { Gabow \& Tarjan } \\
\text { Orlin \& Ahuja } \\
\text { Goldberg \& Kennedy } \\
\text { Duan \& Su }\end{array}$ & INTEGER WEIGHTS \\
\hline 1996 & Cheriyan \& Mehlhorn & $n^{5 / 2} \log (n N)\left(\frac{\log \log n}{\log n}\right)^{1 / 4}$ \\
\hline 1999 & Kao, Lam, Sung \& Ting & $\begin{array}{lr}N m \sqrt{n} / \kappa & \text { MWM ONLY, INTEGER WEIGHTS } \\
N\left(n^{2}+n^{5 / 2} / w\right) & \text { MWM ONLY, INTEGER WEIGHTS } \\
N n^{\omega} & \text { MWM ONLY, RANDOMIZED, INTEGER WEIGHTS }\end{array}$ \\
\hline 2006 & Sankowski & RANDOMIZED, INTEGER WEIGHTS \\
\hline 2012 & Duan \& Su & MWM ONLY, INTEGER WEIGHTS \\
\hline
\end{tabular}

Note: $N$ is the maximum integer edge weight, $w$ is the machine word size, and $\kappa=$ $\log n / \log \left(n^{2} / m\right)$. The time bounds of Johnson [1975] and Fredman and Tarjan [1987] reflect faster priority queues. The time bounds of Kao et al. [2001] reflect a reduction from MWM to $N$ instances of MCM.

path computations on a nonnegatively weighted directed graph. ${ }^{3}$ Using Fibonacci heaps, $n$ executions of Dijkstra's [1959] shortest path algorithm take $O\left(m n+n^{2} \log n\right)$ time. On integer weighted graphs this algorithm can be implemented slightly faster, in $O\left(m n+n^{2} \log \log n\right)$ time [Han 2002; Thorup 2003] or $O(m n)$ time (randomized) [Andersson et al. 1998; Thorup 2007], independent of the maximum edge weight. Gabow and Tarjan [1989], improving an earlier algorithm of Gabow [1985b], gave a scaling algorithm for the assignment problem running in $O(m \sqrt{n} \log (n N))$ time, which is just a $\log (n N)$ factor slower than the fastest MCM algorithm [Hopcroft and Karp 1973; Karzanov 1973]. ${ }^{4}$ For reasonably sparse graphs Gabow and Tarjan's [1989] assignment algorithm remains unimproved. However, faster algorithms have been developed when $N$ is small or the graph is dense [Cheriyan and Mehlhorn 1996; Kao

\footnotetext{
${ }^{3}$ It was known that the assignment problem is reducible to $n$ shortest path computations on arbitrarily weighted graphs. See Ford and Fulkerson [1962], Hoffman and Markowitz [1963], and Desler and Hakimi [1969] for different reductions.

${ }^{4}$ Gabow and Tarjan's algorithm takes a Hungarian-type approach. The same time bound has been achieved by Orlin and Ahuja [1992] using the auction approach of Bertsekas [1981], by Goldberg and Kennedy [1997] using a preflow-push approach, and by Duan and Su [2012] using a primal cycle-canceling approach.
} 
Table III. Weighted Matching: General Graphs

\begin{tabular}{|c|c|c|}
\hline Year & Authors & Time Bound \& Notes \\
\hline 1965 & Edmonds & $m n^{2}$ \\
\hline 1974 & Gabow & \multirow{2}{*}{$n^{3}$} \\
\hline 1976 & Lawler & \\
\hline 1976 & Karzanov & $n^{3}+m n \log n$ \\
\hline 1978 & Cunningham \& Marsh & $\operatorname{poly}(n)$ \\
\hline 1982 & Galil, Micali \& Gabow & $m n \log n$ \\
\hline 1985 & Gabow & INTEGER WEIGHTS \\
\hline & & $N m \sqrt{n} \quad$ MWM ONLY, INTEGER WEIGHTS \\
\hline 1989 & Gabow, Galil \& Spencer & $m n \log \log \log _{d} n+n^{2} \log n \quad d=2+m / n$ \\
\hline 1990 & Gabow & $m n+n^{2} \log n$ \\
\hline 1991 & Gabow \& Tarjan & $m \sqrt{n \log n} \log (n N)$ \\
\hline 2012 & Huang \& Kavitha & \multirow{2}{*}{$\begin{array}{l}N m \sqrt{n} / \kappa \quad \text { MWM ONLY, INTEGER WEIGHTS } \\
N n^{\omega} \quad \text { MWM ONLY, RANDOMIZED, INTEGER WEIGHTS }\end{array}$} \\
\hline 2012 & Pettie & \\
\hline 2012 & $\begin{array}{l}\text { Cygan, Gabow } \\
\text { \& Sankowski }\end{array}$ & $\mathrm{Nn}^{\omega} \quad$ RANDOMIZED, INTEGER WEIGHTS \\
\hline
\end{tabular}

Note: $N$ is the maximum integer edge weight, $\omega$ is the exponent of $n \times n$ matrix multiplication, and $\kappa=\log n / \log \left(n^{2} / m\right)$. The bounds of Huang \& Kavitha and Pettie reflect a reduction from MWM to $N$ instances of MCM.

Table IV. Approximate Maximum Cardinality/Weight Matching

\begin{tabular}{|c|l|l|l|}
\hline Year & \multicolumn{1}{|c|}{ Authors } & Approx. Problem & \multicolumn{2}{|c|}{ Time Bound \& Notes } \\
\hline 1971 & Hopcroft \& Karp & $(1-\epsilon)$-MCM & $m \epsilon^{-1}$ \\
1973 & Dinic \& Karzanov & & $m \epsilon^{-1}$ \\
\hline 1980 & Micali \& Vazirani & $(1-\epsilon)$-MCM & ${ }^{-1}$ \\
1991 & Gabow \& Tarjan & & \\
\hline
\end{tabular}

\begin{tabular}{|c|c|c|c|}
\hline & folklore/trivial & $\frac{1}{2}$-MWM & $m \log n$ \\
\hline 1988 & Gabow \& Tarjan & $(1-\epsilon)-\mathrm{MWM}$ & $m \sqrt{n} \log (n / \epsilon)$ \\
\hline 1991 & Gabow \& Tarjan & $(1-\epsilon)-\mathrm{MWM}$ & $m \sqrt{n \log n} \log (n / \epsilon)$ \\
\hline $\begin{array}{l}1999 \\
2003\end{array}$ & $\begin{array}{l}\text { Preis } \\
\text { Drake \& Hougardy }\end{array}$ & $\frac{1}{2}$-MWM & $m$ \\
\hline 2003 & Drake \& Hougardy & $\left(\frac{2}{3}-\epsilon\right)-\mathrm{MWM}$ & $m \epsilon^{-1}$ \\
\hline 2004 & Pettie \& Sanders & $\left(\frac{2}{3}-\epsilon\right)-\mathrm{MWM}$ & $m \log \epsilon^{-1}$ \\
\hline $\begin{array}{l}2010 \\
2010\end{array}$ & $\begin{array}{l}\text { Duan \& Pettie } \\
\text { Hanke \& Hougardy }\end{array}$ & $\left(\frac{3}{4}-\epsilon\right)-\mathrm{MWM}$ & $m \log n \log \epsilon^{-1}$ \\
\hline & new & $(1-\epsilon)-\mathrm{MWM}$ & $\begin{array}{lr}m \epsilon^{-1} \log \epsilon^{-1} & \text { ARBITRARY WEIGHTS } \\
m \epsilon^{-1} \log N & \text { INTEGER WEIGHTS }\end{array}$ \\
\hline
\end{tabular}

Note: $N$ is the maximum integer edge weight and $\epsilon>0$ is arbitrary.

et al. 2001; Sankowski 2009], or when an MWM is sought [Duan and Su 2012], or when a matching of a specified size is sought [Ramshaw and Tarjan 2012]. Of particular interest is Sankowski's algorithm [2009], which solves MWPM in $O\left(N n^{\omega}\right)$ time, where $\omega$ is the exponent of square matrix multiplication. 


\subsection{Algorithms for General Graphs}

Whereas the basic solutions to $(1,3)$ are integral on bipartite graphs, the same is not true for general graphs. For example, if the graph is a unit-weighted cycle with length $2 k+1$ the MWM has weight $k$ but (1) achieves its maximum of $k+1 / 2$ by setting $x(e)=1 / 2$ for all $e \in E$. Let $\mathcal{V}_{\text {odd }}$ be the set of all odd-size subsets of $V$. Clearly every feasible solution to the integer linear program $(1,2)$ also satisfies the following odd-set constraints.

$$
\sum_{e \in E(B)} x(e) \leq(|B|-1) / 2 \quad \forall B \in \mathcal{V}_{\text {odd }}
$$

Edmonds [1965a, 1965b] proved that if we replace the integrality constraint (2) with (4), the basic solutions to the resulting LP are integral. ${ }^{5}$ Edmonds' algorithm mimics the structure of the Hungarian algorithm but the search for augmenting paths is complicated by the presence of odd-length alternating cycles and the fact that matched edges must be searched in both directions. Edmonds' solution is to contract blossoms as they are encountered. A blossom is defined inductively as an odd-length cycle alternating between matched and unmatched edges, whose components are either single vertices or blossoms in their own right. Blossoms are discussed in detail in Section 2.1.

The fastest implementation of Edmonds' algorithm, due to Gabow [1990], runs in $O\left(m n+n^{2} \log n\right)$ time, which matches the running time of the best bipartite MWPM algorithm [Fredman and Tarjan 1987]. Gabow and Tarjan [1991] extended their scaling algorithm for MWPM to general graphs, achieving a running time of $O(m \sqrt{n \log n} \log (n N))$, which is the fastest known algorithm for integer-weighted graphs and nearly matches the $O(m \sqrt{n})$ time bound of the best MCM algorithms [Micali and Vazirani 1980; Vazirani 1994]. ${ }^{6}$ As in the bipartite case, faster algorithms for MWM and MWPM are known when the graph is dense or $N$ is small. Huang and Kavitha [2012] and Pettie [2012] gave reductions from MWM to $N$ instances of MCM, which can be used in conjunction with the MCM algorithms of Mucha and Sankowski [2004], Harvey [2009], and Goldberg and Karzanov [2004]. Cygan et al. [2012] recently showed that MWPM on general graphs is solvable in $O\left(N n^{\omega}\right)$ time, matching the time bound of Sankowski [2009] for bipartite graphs.

\subsection{Approximating Weighted Matching}

The approximate MWM problem is remarkable in that it has been studied for decades, has practical applications, and yet as late as 1999, essentially nothing better than the greedy algorithm was known. ${ }^{7}$ Moreover, the $(1-\epsilon)$-MCM problem had been solved satisfactorily in the early $1970 \mathrm{~s}$. Although not stated as such, the $O(m \sqrt{n})$-time exact MCM algorithms [Dinic 1970; Hopcroft and Karp 1973; Karzanov 1973; Micali and Vazirani 1980] are actually $(1-\epsilon)$-MCM algorithms running in $O\left(m \epsilon^{-1}\right)$ time. These

\footnotetext{
${ }^{5}$ In the MWPM problem $\sum_{e=\left(u, u^{\prime}\right)} x(e)=1$, for all $u \in V$, and we have the freedom to use an alternative variety of odd-set constraints, namely, $\sum_{e=(u, v) \in E: u \in B, v \notin B} x(e) \geq 1, \forall B \in \mathcal{V}_{\text {odd }}$.

${ }^{6}$ Gabow and Tarjan [1991] claim a running time of $O(m \sqrt{n \log n \alpha(m, n)} \log (n N))$, where the $\alpha(m, n)$ factor comes from an $O(m \alpha(m, n))$ implementation of the split-findmin data structure [Gabow 1985a]. This can be reduced to $O(m \log \alpha(m, n))$ [Pettie 2005]. Thorup [1999] noted that on integer-weighted inputs, split-findmin can be implemented in $O(m)$ time on a word-RAM.

${ }^{7}$ The greedy algorithm repeatedly includes the heaviest edge in the matching and removes all incident edges. Gabow and Tarjan [1989, 1991] observed that by retaining the $O(\log (n / \epsilon))$ high-order bits of the edge weights, their exact scaling algorithms become $\tilde{O}(m \sqrt{n})$-time $(1-\epsilon)$-MWM algorithms for bipartite and general graphs.
} 
algorithms are based on three observations (i) a maximal set of shortest augmenting paths can be found in linear time, (ii) augmenting along such a set increases the length of the shortest augmenting path, and (iii) that after $k$ rounds of such augmentations the resulting matching is a $\left(1-\frac{1}{k+1}\right)$-MCM.

Preis [1999] gave a linear-time $\frac{1}{2}$-MWM algorithm, which improves on the greedy algorithm's $O(m \log n)$ running time but not its approximation guarantee. Drake and Hougardy (see Vinkemeier and Hougardy [2005]) presented the first linear time algorithm with an approximation guarantee greater than $1 / 2$. Specifically, they gave a $\left(\frac{2}{3}-\epsilon\right)$-MWM algorithm running in $O\left(m \epsilon^{-1}\right)$ time, for any $\epsilon>0$. The dependence on $\epsilon$ was later improved by Pettie and Sanders [2004]. These algorithms are based on a weighted version of Hopcroft and Karp's [1973] argument, namely that any matching whose weight-augmenting paths and cycles have at least $k$ unmatched edges is necessarily a $\left(1-\frac{1}{k}\right)$-MWM. Duan and Pettie [2010] and Hanke and Hougardy [2010] presented $\left(\frac{3}{4}-\epsilon\right)$-MWM algorithms running in time $O\left(m \log n \log \epsilon^{-1}\right){ }^{8}$

\subsection{New Results}

We present the first $(1-\epsilon)$-MWM algorithm that significantly improves on the $\tilde{O}(m \sqrt{n})$ running times of Gabow and Tarjan [1989, 1991]. Our algorithm runs in $O\left(m \epsilon^{-1} \log \epsilon^{-1}\right)$ time on general graphs and $O\left(m \epsilon^{-1} \cdot \min \left\{\log \epsilon^{-1}, \log N\right\}\right)$ time on integer-weighted general graphs. This is optimal for any fixed $\epsilon$ and near-optimal as a function of $\epsilon$, given the state-of-the-art in MCM algorithms. ${ }^{9}$ Moreover, our algorithm is as simple as one could reasonably hope for. Its search for augmenting paths uses depth first search [Gabow and Tarjan 1991, Sect. 8] rather than the double depth first search of Micali and Vazirani [1980]. It uses no priority queues, split-findmin structures [Gabow 1985a], or the blossom "shells" that arise from Gabow and Tarjan's [1991] scaling technique.

\subsection{Remarks on Approximate Weighted Matching and Its Applications}

Our focus is on algorithms that accept arbitrary input graphs and that give provably good worst-case approximations. These twin objectives are self-evidently attractive, yet nearly all work on approximate weighted matching (prior to Preis [1999]) focused on specialized cases or weaker approximation guarantees. Early work on the problem usually considered complete bipartite graphs, and confirmed the efficiency of heuristics either experimentally or analytically with respect to inputs over some natural distribution [Avis 1978; Brogden 1946; Kuhn and Baumol 1962; Kurtzberg 1962; Motzkin 1956; Thorndike 1950]. See Avis [1983] for a more detailed discussion of heuristics.

Most work in the area considers graphs defined by metrics, often Euclidean metrics. Reingold and Tarjan [1981] proved that the greedy algorithm for metric MWPM ${ }^{10}$ has an approximation ratio of $\approx n^{\log \frac{3}{2}}>n^{0.58}$. Goemans and Williamson [1995] gave a 2-approximation for metric MWPM that can be implemented in $O\left(n^{2}\right)$ time [Gabow and Pettie 2002], or $O\left(m \log ^{2} n\right)$ time [Cole et al. 2001] in metrics defined by $m$-edge

\footnotetext{
${ }^{8}$ Hanke and Hougardy [2010] also claimed a $\left(\frac{4}{5}-\epsilon\right)$-MWM algorithm running in $O\left(m \log ^{2} n \log \epsilon^{-1}\right)$ time, though the details were not substantiated.

${ }^{9}$ Note that any $(1-\epsilon)$-MWM algorithm running in $O(f(\epsilon) m)$ time yields an exact MCM algorithm running in $O(m \cdot(f(\epsilon)+\epsilon n))$ time, for any $\epsilon$. Thus, any $(1-\epsilon)$-MWM algorithm running in $o\left(m \epsilon^{-1}\right)$ time would improve the $O(m \sqrt{n})$ MCM algorithms [Dinic 1970; Hopcroft and Karp 1973; Karzanov 1973; Micali and Vazirani $1980]$.

${ }^{10}$ For metric inputs let MWPM be the minimum-weight perfect matching problem.
} 
graphs. The Euclidean MWPM comes in two flavors: the monochromatic version is given $2 n$ points and the bichromatic version is given $2 n$ points, $n$ of which are colored blue, the rest red, where the matching cannot include monochromatic edges. ${ }^{11}$ Both the mono- and bichromatic variants of 2D Euclidean MWPM can be $(1+\epsilon)$-approximated in $O\left(n \operatorname{poly}\left(\epsilon^{-1}, \log n\right)\right)$ time [Sharathkumar and Agarwal 2012; Varadarajan and Agarwal 1999]. Some work considers the even more specialized case of Euclidean matching in the unit square, which allows for algorithms that guarantee absolute upper bounds on the weight of the matching; see Iri et al. [1983], Reingold and Supowit [1983], Avis [1983] and the references therein.

There are several applications of MWM (on general or bipartite graphs) in which one would gladly sacrifice matching quality for speed. In input-queued switches packets are routed across a switch fabric from input to output ports. In each cycle one partial permutation can be realized. Existing algorithms for choosing these matchings, such as iSLIP [McKeown 1999] and PIM [Anderson et al. 1993], guarantee $\frac{1}{2}$-MCMs and it has been shown [Giaccone et al. 2005; McKeown et al. 1996] that (approximate) MWMs have good throughput guarantees, where edge weights are based on queue-length. See also Leonardi et al. [2003], Shah and Kopikare [2002], and Shah et al. [2002]. Approximate MWM algorithms are a component in several multilevel graph clustering libraries. $^{12}$ (PARTY, for example, builds a hierarchical clustering by iteratively finding and contracting approximate MWMs; see Preis and Diekmann [1997].) Approximate MWM algorithms are used as a heuristic preprocessing step in several sparse linear system solvers [Duff and Gilbert 2002; Hagemann and Schenk 2006; Olschowka and Neumaier 1996; Schenk et al. 2007]. The goal is to permute the rows/columns to maximize the weight on or near the main diagonal.

\subsection{Organization}

Section 2 introduces some notation, states well-known properties of augmenting paths and blossoms, and reviews Edmonds' optimality conditions for weighted matching. In Section 3, we present our $(1-\epsilon)$-MWM algorithms.

\section{PRELIMINARIES}

We use $E(H)$ and $V(H)$ to refer to the edge and vertex sets of $H$ or the graph induced by $H$, that is, $V\left(E^{\prime}\right)$ is the set of endpoints of $E^{\prime} \subseteq E$ and $E\left(V^{\prime}\right)$ is the edge set of the graph induced by $V^{\prime} \subseteq V$. A matching $M$ is a set of vertex-disjoint edges. Vertices not incident to an $M$ edge are free. An alternating path (or cycle) is one whose edges alternate between $M$ and $E \backslash M$. An alternating path $P$ is augmenting if $P$ begins and ends at free vertices, that is, $M \oplus P \stackrel{\text { def }}{=}(M \backslash P) \cup(P \backslash M)$ is a matching with cardinality $|M \oplus P|=|M|+1$.

Because we are only seeking a $(1-\epsilon)$-approximate solution, we can afford to scale and round edge weights to small integers. To see this, observe that the weight of the MWM is at least $w_{\max }=\max \{w(e) \mid e \in E(G)\}$. It suffices to find a $(1-\epsilon / 2)$-MWM $M$ with respect to the weight function $\tilde{w}(e)=\lfloor w(e) / \gamma\rfloor$ where $\gamma=\epsilon \cdot w_{\max } / n$. Note that

\footnotetext{
${ }^{11}$ The weight of the bichromatic MWPM is also known as the earth mover distance between the red and blue points.

${ }^{12}$ For example, METIS [Karypis and Kumar 1998], PARTY [Preis and Diekmann 1997], PT-SCOTCH [Pellegrini 2008] CHACO [Hendrickson and Leland 1995], JOSTLE [Walshaw and Cross 2007], and $\mathrm{KaFFPa} / \mathrm{KaFFPaE}$ [Holtgrewe et al. 2010; Sanders and Schulz 2012].
} 
$w(e)-\gamma<\gamma \cdot \tilde{w}(e) \leq w(e)$ for any $e$. Define $M^{*}$ and $\tilde{M}^{*}$ to be the MWMs with respect to $w$ and $\tilde{w}$. It follows that:

$$
\begin{aligned}
w(M) & \geq \gamma \cdot \tilde{w}(M) \\
& \geq \gamma \cdot(1-\epsilon / 2) \cdot \tilde{w}\left(\tilde{M}^{*}\right) \\
& \geq \gamma \cdot(1-\epsilon / 2) \cdot \tilde{w}\left(M^{*}\right) \\
& >\gamma \cdot(1-\epsilon / 2) \cdot\left(\gamma^{-1} \cdot w\left(M^{*}\right)-\left|M^{*}\right|\right) \\
& \geq(1-\epsilon / 2) \cdot\left(w\left(M^{*}\right)-\gamma n / 2\right) \\
& =(1-\epsilon / 2) \cdot\left(w\left(M^{*}\right)-\epsilon \cdot w_{\max } / 2\right) \\
& >(1-\epsilon) \cdot w\left(M^{*}\right)
\end{aligned}
$$

Defn. of $\tilde{w}$

Defn. of $M$

Defn. of $\tilde{M}^{*}$

Defn. of $\tilde{w}$

$\left|M^{*}\right| \leq n / 2$

Defn. of $\gamma$

$w\left(M^{*}\right) \geq w_{\max }$.

Since it is better to use an exact MWM algorithm when $\epsilon<1 / n$ [Gabow 1990; Gabow and Tarjan 1991], we assume, henceforth, that $w: E \rightarrow\{1,2, \ldots, N\}$, where $N \leq n^{2}$ is the maximum integer edge weight. For notational convenience, we also assume that $N$ is a power of 2 .

\subsection{Blossoms and the LP Formulation of MWM}

The dual LP of $(1,4)$ is

$$
\begin{array}{lll}
\text { minimize } & \sum_{u \in V(G)} y(u)+\sum_{B \in \mathcal{V}_{\text {odd }}} \frac{|B|-1}{2} \cdot z(B) & \\
\text { subject to } & y z(e) \geq w(e) & \\
& y(u) \geq 0, z(B) \geq 0 & \forall e \in E(G) \\
& \forall u \in V(G), \forall B \in \mathcal{V}_{\text {odd }}
\end{array}
$$

where, by definition, $\quad y z(u, v) \stackrel{\text { def }}{=} y(u)+y(v)+\sum_{\substack{B \in \mathcal{V}_{\text {odd }} \text {, } \\(u, v) \in E(B)}} z(B)$.

Note that $y$ and $z$ map vertices and odd sets to their dual values. It is convenient to create a dual $y z(e)$ for each edge $e$, defined to be the sum of the $y$-values of its endpoints and the $z$-values of all odd sets that contain $e$.

Despite the exponential number of primal constraints and dual $z$-variables, Edmonds [1965a] demonstrated that an optimum matching could be found in polynomial time by maintaining information ( $z$-values) on no more than $n / 2$ elements of $\mathcal{V}_{\text {odd }}$ at any given time. At intermediate stages of Edmonds' algorithm [1965a], there is a matching $M$ and a laminar (nested) subset $\Omega \subseteq \mathcal{V}_{\text {odd }}$ of blossoms. A blossom is identified with a vertex set $B \in \mathcal{V}_{\text {odd }}$ and an edge set $E_{B}$ on $B$. If $v \in V$, then $B=\{v\}$ is a trivial blossom with $E_{B}=\emptyset$. Suppose there is an odd-length sequence of blossoms $A_{0}, A_{1}, \ldots, A_{\ell}$ with associated edge sets $E_{A_{0}}, \ldots, E_{A_{\ell}}$. If the $\left\{A_{i}\right\}$ are connected in a cycle by edges $e_{0}, \ldots, e_{\ell}$, where $e_{i} \in A_{i} \times A_{i+1}$ (modulo $\ell+1$ ), then $B=\bigcup_{i} A_{i}$ is also a blossom associated with edge set $E_{B}=\bigcup_{i} E_{A_{i}} \cup\left\{e_{0}, \ldots, e_{\ell}\right\}$. A short proof by induction shows that $|B|$ is odd. A blossom $B$ is full with respect to a matching $M$ if $\left|M \cap E_{B}\right|=(|B|-1) / 2$, that is, $M \cap E_{B}$ matches all vertices except one, which is called the base of $B$. Only full blossoms are included in $\Omega$. Note that $E(B)=E \cap(B \times B)$ may contain many edges outside of $E_{B}$.

A key property of blossoms is that any vertex $b \in B$ can be made the base by choosing a suitable matching $M$ on $E_{B}$. Suppose, without loss of generality, that $b \in A_{0}$ and let $e_{i}=\left(u_{i}, v_{i+1}\right)$ (modulo $\ell+1$ ), where $u_{i}, v_{i} \in A_{i}$. Recursively find matchings in $\left\{E_{A_{i}}\right\}$ using $b$ as the base of $A_{0}, u_{i}$ as the base of $A_{i}$ when $i$ is odd, and $v_{i}$ as the base of $A_{i}$ when $i>0$ is even. At this point the only unmatched vertices are the bases 


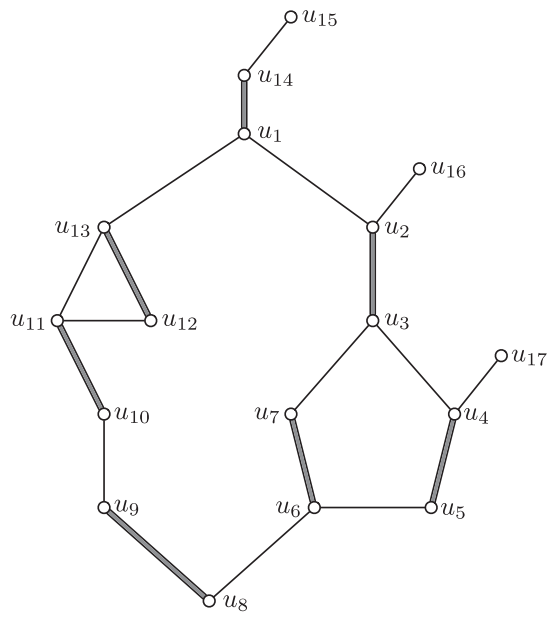

(a)

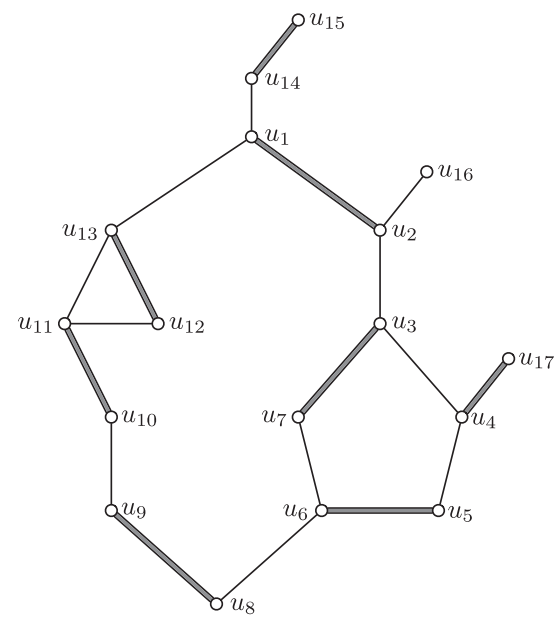

(b)

Fig. 1. Thick edges are matched, thin unmatched. (a) A blossom $B_{1}=\left(u_{1}, u_{2}, B_{2}, u_{8}, u_{9}, u_{10}, B_{3}\right)$ with base $u_{1}$ containing nontrivial sub-blossoms $B_{2}=\left(u_{3}, u_{4}, u_{5}, u_{6}, u_{7}\right)$ with base $u_{3}$ and $B_{3}=$ $\left(u_{11}, u_{12}, u_{13}\right)$ with base $u_{11}$. Vertices $u_{15}, u_{16}$, and $u_{17}$ are free. The path $\left(u_{16}, u_{2}, u_{3}, u_{7}, u_{6}, u_{5}, u_{4}, u_{17}\right)$ is an example of an augmenting path that exists in $G$ but not $G / B_{1}$, the graph obtained by contracting $B_{1}$. (b) The situation after augmenting along $\left(u_{15}, u_{14}, B_{1}, u_{17}\right)$ in $G / B_{1}$, which corresponds to augmenting along $\left(u_{15}, u_{14}, u_{1}, u_{2}, u_{3}, u_{7}, u_{6}, u_{5}, u_{4}, u_{17}\right)$ in $G$. After augmentation $B_{1}$ and $B_{2}$ have their base at $u_{4}$. If, instead, we augmented along $\left(u_{15}, u_{14}, B_{1}, u_{16}\right)$, corresponding to the path $\left(u_{15}, u_{14}, u_{1}, u_{13}, u_{12}, u_{11}, u_{10}, u_{9}, u_{8}, u_{6}, u_{7}, u_{3}, u_{2}, u_{16}\right)$, the base of $B_{1}$ would be relocated to $u_{2}$ and the bases of $B_{2}$ and $B_{3}$ would be relocated to $u_{6}$ and $u_{13}$, respectively.

$\{b\} \cup\left\{u_{i}\right\}_{i \text { odd }} \cup\left\{v_{i}\right\}_{i \text { even. }}$. Include in the matching the edges $\left\{\left(u_{i}, v_{i+1}\right)\right\}_{i \text { odd }}$, which leaves only $b$ unmatched. (See Figure 1.) The blossom structure guarantees that there is an even-length alternating path from $b$ to every other vertex $c$ in $B$. Inductively assume this claim holds for $A_{0}, \ldots, A_{\ell}$. Suppose $c \in A_{i}$. If $i$ is even, take a path from $b$ to $c$ via edges $\left(u_{0}, v_{1}\right),\left(u_{1}, v_{2}\right), \ldots,\left(u_{i-1}, v_{i}\right)$. The inductive hypothesis gives us even-length alternating paths from $b$ to $u_{0}$ in $E_{A_{0}}$, from $v_{1}$ to $u_{1}$ in $E_{A_{1}}$, and so on. If $i$ is odd, we go around the cycle in the other direction, taking a path from $b$ to $c$ via edges $\left(v_{0}, u_{\ell}\right),\left(v_{\ell}, u_{\ell-1}\right), \ldots,\left(v_{i+1}, u_{i}\right)$. (See Figure 1.)

Matching algorithms represent a nested set $\Omega$ of full, active blossoms by rooted trees, where leaves represent vertices and internal nodes represent nontrivial blossoms. A root blossom is one not contained in any other blossom. The children of an internal node representing $B$ are ordered according to the odd cycle that formed $B$, where one child is distinguished as containing the base of $B$. As we will see, it is often possible to treat blossoms as if they were single vertices. Let the contracted graph $G / \Omega$ be obtained by contracting all root blossoms and removing spurious edges. To dissolve a root blossom $B$ means to delete its node in the blossom forest and, in the contracted graph, to replace $B$ with individual vertices $A_{0}, \ldots, A_{\ell}$. Lemma 2.1 summarizes some well-known properties of blossoms and the contracted graph.

LEMMA 2.1. Let $\Omega$ be a set of full blossoms with respect to a matching $M$ and let $B \in \Omega$ be a root blossom.

(1) If $M$ is a matching in $G$, then $M / \Omega$ is a matching in $G / \Omega$.

(2) Every augmenting path $P^{\prime}$ relative to $M / \Omega$ in $G / \Omega$ extends to an augmenting path $P$ relative to $M$ in $G$. (That is, $P$ is obtained from $P^{\prime}$ by substituting for each nontrivial blossom vertex $B$ in $P^{\prime}$ a path through $E_{B}$. See Figure 1.) 
(3) Let $P^{\prime}$ and $P$ be as mentioned in (2). Then, $\Omega$ remains a valid set of full blossoms (possibly with different bases) with respect to the augmented matching $M \oplus P$. (See Figure 1.)

Proof. Part (1) follows from the fact that $M \cap E_{B}$ leaves only one vertex in $B$ unmatched, namely its base, implying $B$ is incident to at most one edge in $G / \Omega$. For Part (2) consider an augmenting path $P^{\prime}=\left(B_{0}, B_{1}, \ldots, B_{k}\right)$ in $G / \Omega$. The matched edge in $G$ corresponding to $\left(B_{i}, B_{i+1}\right)$, where $i$ is odd, necessarily connects the bases of $B_{i}$ and $B_{i+1}$. We can therefore extend $P^{\prime}$ to an augmenting path $P$ in $G$ by substituting for each $B_{i}$ a suitable even-length alternating path through $E_{B_{i}}$, one endpoint of which is the base of $B_{i}$. Turning to Part (3), suppose $P$ enters a blossom $B$ at its base $b$ and leaves at a vertex $c$. It follows that $b$ is either free or matched to a vertex outside of $B$. After augmentation $b$ will be matched and $c$ will be matched to a vertex outside of $B$, that is, augmenting along $P$ shifts the base from $b$ to $c$. Augmenting along $P$ may also shift the bases of other blossoms contained in $B$. For example, suppose $b \in A_{0}, c \in A_{i}$, and $i$ is even. (As defined earlier, $B$ is composed of sub-blossoms $\left\{A_{i}\right\}$ connected by cycle edges $\left\{\left(u_{i}, v_{i+1}\right)\right\}$.) The portion of $P$ in $E_{B}$ traverses cycle edges $\left(u_{0}, v_{1}\right), \ldots,\left(u_{i-1}, v_{i}\right)$ and even-length alternating paths in each of $A_{0}, A_{1}, \ldots, A_{i}$; augmenting along $P$ relocates all of their bases. For example, the base of $A_{0}$ is relocated from $b$ to $u_{0}$ and the base of $A_{1}$ from $u_{1}$ to $v_{1}$, since $\left(u_{0}, v_{1}\right) \in M \oplus P$.

Implementations of Edmonds' algorithm grow a matching $M$ while maintaining Property 2.2, which controls the relationship between $M, \Omega$ and the dual variables.

\section{Property 2.2. (Feasibility and Complementary Slackness Conditions)}

(1) Nonnegativity. $z(B) \geq 0$ for all $B \in \mathcal{V}_{\text {odd }}$ and $y(u) \geq 0$ for all $u \in V(G)$. If $y(u)>0$, then $u$ is matched.

(2) Active Blossoms. $\Omega$ contains all $B$ with $z(B)>0$ and all root blossoms $B$ have $z(B)>0$. (Non-root blossoms may have zero $z$-values.)

(3) Domination. $y z(e) \geq w(e)$ for all $e \in E$.

(4) Tightness. $y z(e)=\bar{w}(e)$ when $e \in M$ or $e \in E_{B}$ for some $B \in \Omega$.

If the $y$-values of free vertices become zero, it follows from domination and tightness that $M$ is a maximum weight matching, as the following short proof attests. Here $M^{*}$ is any maximum weight matching.

$$
\begin{array}{rlr}
w(M) & =\sum_{e \in M} w(e)=\sum_{e \in M} y z(e) & \text { tightness } \\
& =\sum_{u \in V(G)} y(u)+\sum_{B \in \Omega} \frac{|B|-1}{2} \cdot z(B) & \text { free } y \text {-values, defn. of } y z \\
& \geq \sum_{u \in V\left(M^{*}\right)} y(u)+\sum_{B \in \Omega}\left|E(B) \cap M^{*}\right| \cdot z(B) & y, z \text { nonnegative } \\
& =\sum_{e \in M^{*}} y z(e) \geq w\left(M^{*}\right) & \text { domination }
\end{array}
$$

Our approximate MWM algorithms are based on the observation that if domination and tightness are satisfied up to a $1 \pm \epsilon$ factor, then the given matching is a $(1-O(\epsilon))$ MWM.

LEMMA 2.3. Let $M$ and $\Omega$ be a matching and set of nested blossoms, and let $y$ and $z$ assign their dual values. Suppose y, $z$ satisfy Property $2.2(1,2)$ and satisfy Property $2.2(3,4)$ in the following approximate sense. For all e, $y z(e) \geq\left(1-\epsilon_{0}\right) \cdot w(e)$, and for 
all $e \in M \cup \cup_{B \in \Omega} E_{B}, y z(e) \leq\left(1+\epsilon_{1}\right) \cdot w(e)$. If the $y$-values of free vertices are zero, $M$ is $a\left(\left(1+\epsilon_{1}\right)^{-1}\left(1-\epsilon_{0}\right)\right)$-MWM.

PROOF. From the definitions of approximate tightness and approximate domination, we have the following.

$$
\begin{aligned}
w(M) & =\sum_{e \in M} w(e) \geq\left(1+\epsilon_{1}\right)^{-1} \sum_{e \in M} y z(e) \\
& =\left(1+\epsilon_{1}\right)^{-1}\left(\sum_{u \in V(G)} y(u)+\sum_{B \in \Omega} \frac{|B|-1}{2} \cdot z(B)\right) \\
& \geq\left(1+\epsilon_{1}\right)^{-1}\left(\sum_{u \in V\left(M^{*}\right)} y(u)+\sum_{B \in \Omega}\left|E(B) \cap M^{*}\right| \cdot z(B)\right) \\
& =\left(1+\epsilon_{1}\right)^{-1} \cdot \sum_{e \in M^{*}} y z(e) \\
& \geq\left(1+\epsilon_{1}\right)^{-1}\left(1-\epsilon_{0}\right) \cdot w\left(M^{*}\right)
\end{aligned}
$$

approx. tightness

free $y$-values, defn. of $y z$

$y, z$ nonnegative

defn. of $y z$

approx. domination

\section{A SCALING ALGORITHM FOR APPROXIMATE MWM}

Our algorithm maintains a dynamic relaxation of the feasibility and complementary slackness conditions. In the beginning, domination is weak but becomes progressively tighter at each scale whereas tightness is weakened at each scale, though not uniformly. The degree to which a matched edge or blossom edge may violate tightness depends on the scale when it last entered the blossom or matching.

Recall that $N$ is the maximum integer edge weight. The parameter $\epsilon^{\prime}=\Theta(\epsilon)$ will be selected later to guarantee that the final matching is a $(1-\epsilon)$-MWM. Henceforth, assume that $N \geq 1$ and $\epsilon^{\prime} \leq 1 / 4$ are powers of two. Define $\delta_{0}=\epsilon^{\prime} N$ and $\delta_{i}=\delta_{0} / 2^{i}$. At scale $i$ we use the truncated weight function $w_{i}(e)=\delta_{i}\left\lfloor w(e) / \delta_{i}\right\rfloor$. Note that $w_{i+1}(e)=$ $w_{i}(e)$ or $w_{i}(e)+\delta_{i+1}$.

Property 3.1. (Relaxed Feasibility and Complementary Slackness). There are $L+1$ scales numbered $0, \ldots, L$, where $L=\log N$. Let $i \in[0, L]$ be the current scale.

(1) Granularity. $z(B)$ is a nonnegative multiple of $\delta_{i}$, for all $B \in \mathcal{V}_{\text {odd }}$, and $y(u)$ is a nonnegative multiple of $\delta_{i} / 2$, for all $u \in V(G)$.

(2) Active Blossoms. $\Omega$ contains all $B$ with $z(B)>0$ and all root blossoms $B$ have $z(B)>0$. (Non-root blossoms may have zero $z$-values.)

(3) Near Domination. $y z(e) \geq w_{i}(e)-\delta_{i}$ for all $e \in E$.

(4) Near Tightness. Call a matched or blossom edge type $j$ if it was last made a matched or blossom edge in scale $j \leq i$. (That is, it entered the set $M \cup \cup_{B \in \Omega} E_{B}$ in scale $j$ and has remained in that set, even as $M$ and $\Omega$ evolve as augmenting paths are found and blossoms are formed and dissolved.) If $e$ is such a type $j$ edge, then $y z(e) \leq w_{i}(e)+2\left(\delta_{j}-\delta_{i}\right)$.

(5) Free Vertex Duals. The $y$-values of free vertices are equal and strictly less than the $y$-values of matched vertices.

Property 3.1's definitions of near domination and near tightness differ from their analogues in previous (exact) scaling algorithms. The differences stem from the halting conditions for a scale and how dual variables are adjusted between scales. In Gabow and Tarjan's [1991] algorithm, for example, scale $i$ begins by forgetting the matching 
and blossom edges from the previous scale (though not the nested structure of the old blossoms), replacing the weight function $w_{i-1}$ by $w_{i}$, and adjusting the duals to guarantee domination with respect to $w_{i} \cdot{ }^{13}$ Since the matching and blossom edges are forgotten, near tightness holds trivially. The scale ends once the algorithm finds a perfect matching, blossoms, and duals $y, z$ that satisfy tightness to within $\delta_{i}$.

For subsequent scales of the Gabow-Tarjan algorithm to be efficient, it is critical that each scale ends with a perfect matching and nearly optimum duals. Our algorithm, however, is obliged to run in linear time (for fixed $\epsilon$ ) and therefore cannot afford to find a perfect matching, nor can it afford to discard the matching computed by one scale before proceeding to the next. The goal of a scale must be different. Consider the difficulty of preserving Property 3.1(3,4) (near tightness and near domination) across multiple scales. At the end of scale $i-1$, we have $y z(e) \geq w_{i-1}(e)-\delta_{i-1}$ whereas at the beginning of scale $i$ we require $y z(e) \geq w_{i}(e)-\delta_{i}$. We force near domination to be satisfied by simply having each vertex add $\delta_{i}$ to its $y$-value. However, doing this effectively weakens near-tightness on matched and blossom edges by $2 \delta_{i}$. If an edge $e$ enters the matching at scale $j$ and witnesses $i-j$ scale changes, in scale $i$ it may violate tightness by as much as $2 \delta_{j+1}+\cdots+2 \delta_{i}=2\left(\delta_{j}-\delta_{i}\right)$. The question is whether violations of tightness of this magnitude are tolerable.

In order to invoke Lemma 2.3, we need to write Property 3.1(3,4) as multiplicative $\left(1-\epsilon_{0}\right)$ - and $\left(1+\epsilon_{1}\right)$-approximations, for some $\epsilon_{0}, \epsilon_{1}=O(\epsilon)$. The algorithm halts after scale $L$. At this time $w_{L}=w, \delta_{L}=\epsilon^{\prime}$ and the $y$-values of free vertices are zero. Since weights are positive integers the near dominance condition $y z(e) \geq w_{L}(e)-\delta_{L}=$ $w(e)-\epsilon^{\prime}$ implies $y z(e) \geq\left(1-\epsilon^{\prime}\right) w(e)$. We will show later that the near-tightness condition $y z(e) \leq w_{i}(e)+2\left(\delta_{j}-\delta_{i}\right)$ implies $y z(e) \leq\left(1+\epsilon_{1}\right) w(e)$ for an $\epsilon_{1}=O\left(\epsilon^{\prime}\right)$.

\subsection{The Scaling Algorithm}

Initially, $M=\emptyset, \Omega=\emptyset$, and $y(u)=N / 2-\delta_{0} / 2$ for all $u \in V$, which clearly satisfies Property 3.1 for scale $i=0$, since $y z(e)=2\left(N / 2-\delta_{0} / 2\right) \geq w_{0}(e)-\delta_{0}$. The algorithm, given in Figure 2, consists of scales $0, \ldots, L=\log N$, where the purpose of scale $i$ is to halve the $y$-values of free vertices while maintaining Property 3.1. In each iteration of scale $i$, the algorithm (1) augments a maximal set of vertex-disjoint augmenting paths of eligible edges, (2) finds and contracts blossoms of eligible edges, (3) performs dual adjustments on $y$ - and $z$-values, and (4) dissolves previously contracted root blossoms if their $z$-values become zero. Each dual adjustment step decrements by $\delta_{i} / 2$ the $y$-values of free vertices. Thus, there are roughly $\left(N / 2^{i+2}\right) /\left(\delta_{i} / 2\right)=O\left(\epsilon^{-1}\right)$ iterations per scale, independent of $i$. The efficiency and correctness of the algorithm depend on eligibility being defined properly.

Definition 3.2. At scale $i$, an edge $e$ is eligible if at least one of the following hold.

(i) $e \in E_{B}$ for some $B \in \Omega$.

(ii) $e \notin M$ and $y z(e)=w_{i}(e)-\delta_{i}$.

(iii) $e \in M$ and $y z(e)-w_{i}(e)$ is a nonnegative integer multiple of $\delta_{i}$.

Let $E_{\text {elig }}$ be the set of eligible edges and let $G_{\text {elig }}=\left(V, E_{\text {elig }}\right) / \Omega$ be the unweighted graph obtained by discarding ineligible edges and contracting root blossoms.

Criterion (i) for eligibility simply ensures that an augmenting path in $G_{\text {elig }}$ extends to an augmenting path of eligible edges in $G$. A key implication of Criteria (ii) and (iii) is that if $P$ is an augmenting path in $G_{\text {elig }}$, every edge in $P$ becomes ineligible

\footnotetext{
${ }^{13} \mathrm{By}$ " $w_{i}$ " and " $\delta_{i}$ ", we mean the weight function and granularity used in the $i$ th scale of their algorithm. Their initial granularity $\delta_{0}$ differs from ours.
} 


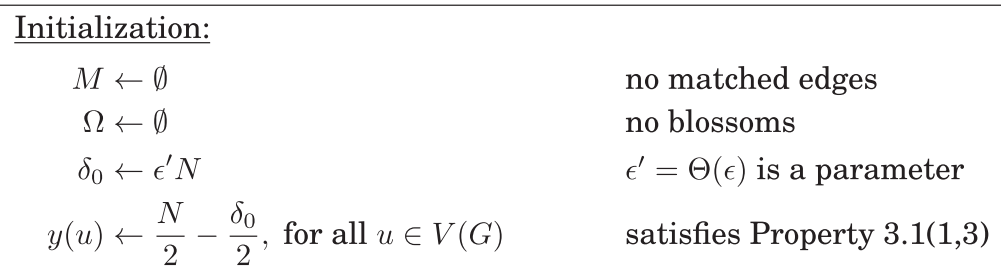

Execute scales $i=0, \ldots, L=\log N$ and return the matching $M$.

Scale $i$ :

- Repeat the following four steps until $y$-values of free vertices reach $N / 2^{i+2}-\delta_{i} / 2$, if $i \in[0, L)$, or until they reach zero, if $i=L$.

(1) Augmentation:

Find a maximal set $\Psi$ of vertex-disjoint augmenting paths in $G_{\text {elig }}$ and set $M \leftarrow M \oplus\left(\bigcup_{P \in \Psi} P\right)$. Update $G_{\text {elig }}$.

(2) Blossom Shrinking:

Let $V_{\text {out }} \subseteq V\left(G_{\text {elig }}\right)$ be the vertices (that is, root blossoms) reachable from free vertices by even-length alternating paths; let $\Omega^{\prime}$ be a maximal set of (nested) blossoms on $V_{\text {out }}$. (That is, if $(u, v) \in E\left(G_{\text {elig }}\right) \backslash M$ and $u, v \in V_{\text {out }}$, then $u$ and $v$ must be in a common blossom.) Let $V_{\text {in }} \subseteq V\left(G_{\text {elig }}\right) \backslash V_{\text {out }}$ be those non- $V_{\text {out }}$-vertices reachable from free vertices by odd-length alternating paths. Set $z(B) \leftarrow 0$ for $B \in \Omega^{\prime}$ and set $\Omega \leftarrow \Omega \cup \Omega^{\prime}$. Update $G_{\text {elig. }}$.

(3) Dual Adjustment:

Let $\hat{V}_{\text {in }}, \hat{V}_{\text {out }} \subseteq V$ be original vertices represented by vertices in $V_{\text {in }}$ and $V_{\text {out }}$. The $y$ - and $z$-values for some vertices and root blossoms are adjusted:

$$
\begin{aligned}
& y(u) \leftarrow y(u)-\delta_{i} / 2, \text { if } u \in \hat{V}_{\text {out }} . \\
& y(u) \leftarrow y(u)+\delta_{i} / 2, \text { if } u \in \hat{V}_{\text {in }} . \\
& z(B) \leftarrow z(B)+\delta_{i}, \text { if } B \in \Omega \text { is a root blossom with } B \subseteq \hat{V}_{\text {out }} . \\
& z(B) \leftarrow z(B)-\delta_{i}, \text { if } B \in \Omega \text { is a root blossom with } B \subseteq \hat{V}_{\text {in }} .
\end{aligned}
$$

(4) Blossom Dissolution:

After dual adjustments some root blossoms may have zero $z$-values. Dissolve such blossoms (remove them from $\Omega$ ) as long as they exist. Note that non-root blossoms are allowed to have zero $z$-values. Update $G_{\text {elig }}$ by the new $\Omega$.

- Prepare for the next scale, if $i \in[0, L)$ :

$$
\begin{aligned}
& \delta_{i+1} \leftarrow \delta_{i} / 2 \\
& y(u) \leftarrow y(u)+\delta_{i+1}, \text { for all } u \in V(G)
\end{aligned}
$$

free vertices' $y$-values are $N / 2^{i+2}$.

Fig. 2. The scaling algorithm.

after augmentation. This follows from the fact that eligible unmatched edges must have $y z(e)-w_{i}(e)<0$ whereas eligible matched edges must have $y z(e)-w_{i}(e) \geq 0$. Regarding Criterion (iii), note that Property 3.1 (granularity and near domination) implies that $y z(e)-w_{i}(e)$ is at least $-\delta_{i}$ and an integer multiple of $\delta_{i} / 2$. 


\subsection{Analysis and Correctness}

The aim of this section is to prove Lemma 3.5, which states that the algorithm maintains Property 3.1 after each of the $O\left(\epsilon^{-1}\right)$ Dual Adjustment steps in each scale. Lemmas 3.3 and 3.4 establish some facts used in the proof of Lemma 3.5.

LEMma 3.3. After the Augmentation and Blossom Shrinking steps, $G_{\text {elig }}$ contains no augmenting path, nor is there an even-length alternating path from a free vertex to a blossom.

Proof. Suppose there is an augmenting path $P$ in $G_{\text {elig }}$ after augmenting along paths in $\Psi$. Since $\Psi$ is maximal, $P$ must intersect some $P^{\prime} \in \Psi$ at a vertex $v$. However, after the Augmentation step every edge in $P^{\prime}$ will become ineligible, so the matching edge $\left(v, v^{\prime}\right) \in M$ is no longer in $G_{\text {elig }}$, contradicting the fact that $P$ consists of eligible edges. Since $\Omega^{\prime}$ is maximal there can be no blossom reachable from a free vertex in $G_{\text {elig }}$ after the Blossom Shrinking step.

Lemma 3.4 guarantees that all $y$-values updated in a Dual Adjustment step have the same parity as a multiple of $\delta_{i} / 2$, that is, they are either both even or both odd multiples of $\delta_{i} / 2$. In the proof of Lemma 3.5, this fact is used to argue that if both endpoints of an edge $e$ have their $y$-values decremented, then $y z(e)$ is a multiple of $\delta_{i}$.

LEMMA 3.4. Let $R \subseteq V\left(G_{\text {elig }}\right)$ be the set of vertices reachable from free vertices by eligible alternating paths, at any point in scale $i$. Let $\hat{R} \subseteq V(G)$ be the set of original vertices represented by those in $R$. Then, the $y$-values of $\hat{R}$-vertices have the same parity, as a multiple of $\delta_{i} / 2$.

Proof. The claim clearly holds at initialization $(i=0)$ since all vertices have identical $y$-values. Assume, inductively, that before the Blossom Shrinking step, all vertices in a common blossom have the same parity, as a multiple of $\delta_{i} / 2$. (This property is clearly preserved when transitioning from scale $i-1$ to scale $i$.) Consider an eligible path $P=\left(B_{0}, B_{1}, \ldots, B_{k}\right)$ in $G_{e l i g}$, where the $\left\{B_{j}\right\}$ are either vertices or blossoms in $\Omega$ and $B_{0}$ is unmatched in $G_{\text {elig. }}$. Let $\left(u_{0}, v_{1}\right),\left(u_{1}, v_{2}\right), \ldots,\left(u_{k-1}, v_{k}\right)$ be the $G$-edges corresponding to $P$, where $u_{j}, v_{j} \in B_{j}$. By the inductive hypothesis, $u_{j}$ and $v_{j}$ have the same parity, and whether $\left(u_{j}, v_{j+1}\right)$ is matched or unmatched, Definition 3.2 stipulates that $y z\left(u_{j}, v_{j+1}\right) / \delta_{i}$ is an integer, which implies $y\left(u_{j}\right)$ and $y\left(v_{j+1}\right)$ have the same parity as a multiple of $\delta_{i} / 2$. Thus, the $y$-values of all vertices in $B_{0} \cup \ldots \cup B_{k}$ have the same parity as a free vertex in $B_{0}$, whose $y$-value is equal to every other free vertex, by Property 3.1(5). Since new blossoms are formed by eligible edges, the inductive hypothesis is preserved after the Blossom Shrinking step. It is also preserved after the Dual Adjustment step since the $y$-values of vertices in a common blossom are incremented or decremented in lockstep. This concludes the induction.

\section{LEMMA 3.5. The algorithm preserves Property 3.1.}

PROOF. Property 3.1(5) (free vertex duals) is maintained as only free vertices have their $y$-values decremented in each Dual Adjustment step. Any newly matched edge $e$ is ineligible so neither endpoint of $e$ can be in $\hat{V}_{\text {out }}$. Property 3.1(2) (active blossoms) is also maintained since all the new root blossoms discovered in the Blossom Shrinking step are contained in $V_{\text {out }}$ and will have positive $z$-values after adjustment. Furthermore, each root blossom whose $z$-value drops to zero is dissolved, after Dual Adjustment. At the beginning of scale $i$, all $y$ - and $z$-values are integer multiples of $\delta_{i} / 2$ and $\delta_{i}$, respectively, satisfying Property 3.1(1) (granularity). This property is clearly 
maintained in each Dual Adjustment step. If $e \notin M$ is placed in $M$ during an Augmentation step or placed in $\bigcup_{B \in \Omega} E_{B}$ during a Blossom Shrinking step, then $e$ is type $i$ and $y z(e)=w_{i}(e)-\delta_{i}<w_{i}(e)$, which satisfies Property 3.1(4).

It remains to show that the algorithm maintains Property 3.1(3,4) (near domination and near tightness). It is clear that these properties are preserved during Augmentation and Blossom Shrinking. First consider the dual adjustments made at the end of scale $i$ (the last line of pseudocode in Figure 2). Let $e=(u, v)$ be an arbitrary edge and let $y z$ and $y z^{\prime}$ be the function before and after dual adjustment. It follows that

$$
\begin{array}{rlrl}
y z^{\prime}(e) & =y z(e)+2 \delta_{i+1} & y(u), y(v) \text { incremented by } \delta_{i+1} \\
& \geq w_{i}(e)-\delta_{i}+2 \delta_{i+1} & \text { near domination at scale } i \\
& \geq w_{i+1}(e)-\delta_{i+1} & w_{i}(e) \geq w_{i+1}(e)-\delta_{i+1} .
\end{array}
$$

That is, Property 3.1(3) is preserved. If $e \in M \cup \cup_{B \in \Omega} E_{B}$ is a type $j$ edge, then at the end of scale $i$ Property 3.1(4) is also preserved since

$$
y z^{\prime}(e)=y z(e)+2 \delta_{i+1} \leq w_{i}(e)+2\left(\delta_{j}-\delta_{i}\right)+2 \delta_{i+1} \leq w_{i+1}(e)+2\left(\delta_{j}-\delta_{i+1}\right) .
$$

The first inequality follows from Property 3.1(4) at scale $i$ and the second inequality from the fact that $w_{i}(e) \leq w_{i+1}(e)$ and $\delta_{i}=2 \delta_{i+1}$.

Now consider a Dual Adjustment step. If neither $u$ nor $v$ is in $\hat{V}_{\text {in }} \cup \hat{V}_{\text {out }}$ or if $u, v$ are in the same root blossom in $\Omega$, then $y z(e)$ is unchanged, preserving Property 3.1. The remaining cases depend on whether $(u, v)$ is in $M$ or not, whether $(u, v)$ is eligible or not, and whether both $u, v \in \hat{V}_{\text {in }} \cup \hat{V}_{\text {out }}$ or not.

Case 1. $e \notin M, u, v \in \hat{V}_{\text {in }} \cup \hat{V}_{\text {out }}$. If $e$ is ineligible, then $y z(e)>w_{i}(e)-\delta_{i}$. However, by Lemma 3.4 (parity of $y$-values), we know $\left(y z(e)-w_{i}(e)\right) / \delta_{i}$ is an integer, so $y z(e) \geq w_{i}(e)$ before adjustment and $y z(e) \geq w_{i}(e)-\delta_{i}$ afterward (which could occur if both $u, v \in$ $\hat{V}_{\text {out }}$ ), thereby preserving Property 3.1(3). If $e$ is eligible, then at least one of $u, v$ is in $\hat{V}_{i n}$, otherwise, another blossom or augmenting path would have been formed, so $y z(e)$ cannot be reduced, which also preserves Property 3.1(3).

Case 2. $e \in M, u, v \in \hat{V}_{\text {in }} \cup \hat{V}_{\text {out }}$. Since $u, v \in \hat{V}_{\text {in }} \cup \hat{V}_{\text {out }}$, Lemma 3.4 (parity of $y$ values) guarantees that $\left(y z(e)-w_{i}(e)\right) / \delta_{i}$ is an integer. If $y z(e)-w_{i}(e)=-\delta_{i}$, then $e$ is ineligible. Thus, both $u$ and $v$ are in $\hat{V}_{i n}$ and $y z(e)=w_{i}(e)$ after dual adjustment, which preserves Property 3.1(3,4). If $y z(e)-w_{i}(e) \geq 0$, then $e$ is eligible, $u \in \hat{V}_{\text {in }}$, and $v \in \hat{V}_{\text {out }}$. It cannot be that $u, v \in \hat{V}_{\text {out }}$ as otherwise $e$ would have been included in an augmenting path or root blossom. In this case, $y z(e)$ is unchanged by dual adjustment, preserving Property 3.1(3,4).

Case 3. $e \notin M$, only $v \notin \hat{V}_{\text {in }} \cup \hat{V}_{\text {out }}$. If $e$ is eligible, then $u \in \hat{V}_{\text {in }}$ and $y z(e)$ will increase. If it is ineligible, then $y z(e) \geq w_{i}(e)-\delta_{i} / 2$ before adjustment and $y z(e) \geq w_{i}(e)-\delta_{i}$ afterward. In both cases, Property 3.1(3) is preserved.

Case 4. $e \in M$, only $v \notin \hat{V}_{\text {in }} \cup \hat{V}_{\text {out }}$. It must be that $e$ is ineligible, so $u \in \hat{V}_{\text {in }}$ and $y z(e)-w_{i}(e)$ is either negative or an odd multiple of $\delta_{i} / 2$. If $e$ is type $j$, then, by Property $3.1(1,4)$ (granularity and near tightness), $y z(e) \leq w_{i}(e)+2\left(\delta_{j}-\delta_{i}\right)-\delta_{i} / 2$ before adjustment and $y z(e) \leq w_{i}(e)+2\left(\delta_{j}-\delta_{i}\right)$ afterward, preserving Property 3.1(4).

Recall that Lemma 2.3 stated that the final matching will be a $(1-O(\epsilon))$-MWM if free vertices have zero $y$-values, and $|y z(e)-w(e)|=O(\epsilon) \cdot w(e)$. Lemmas 3.6 and 3.7 establish these bounds. 
LEMMA 3.6. Let $i \leq L$ be the scale index. Then

(1) for $i<L$, all edges eligible at any time in scales 0 through $i$ have weight at least $N / 2^{i+1}+\delta_{i}$

(2) for any $i$, if $e \in M$, then $y z(e) \leq\left(1+4 \epsilon^{\prime}\right) w(e)$.

PROOF.

Part 1. The last search for augmenting paths in scale $i$ begins when the $y$-values of free vertices are $N / 2^{i+2}$, and strictly less than $y$-values of other vertices, by Property 3.1(5). An unmatched edge $e=(u, v)$ can only be eligible at this scale if $y z(e)=$ $w_{i}(e)-\delta_{i} \leq w(e)-\delta_{i}$. Hence, $w(e) \geq w_{i}(e) \geq y(u)+y(v)+\delta_{i} \geq N / 2^{i+1}+\delta_{i}$.

Part 2. Let $e$ be a type $j$ edge in $M$ during scale $i$. Property 3.1(4) states that $y z(e)-$ $w_{i}(e) \leq 2\left(\delta_{j}-\delta_{i}\right)$. Since $w_{i}(e) \leq w(e)$ it also follows that $y z(e)-w(e) \leq 2 \delta_{j}-2 \delta_{i}<$ $2 \delta_{j}=\epsilon^{\prime} N / 2^{j-1}$. By part 1 , a type $j$ edge must have weight at least $N / 2^{j+1}+\delta_{j}$, hence $y z(e)-w(e)<4 \epsilon^{\prime} \cdot w(e)$.

LEMMA 3.7. After scale $L=\log N$, $M$ is a $\left(1-5 \epsilon^{\prime}\right)$-MWM.

PRoof. The final scale ends when free vertices have zero $y$-values and $\delta_{L}=\epsilon^{\prime}$. According to Lemmas 3.6 and 2.3, $w(M) \geq\left(1-\epsilon^{\prime}\right)\left(1+4 \epsilon^{\prime}\right)^{-1} \cdot w\left(M^{*}\right)>\left(1-5 \epsilon^{\prime}\right) \cdot w\left(M^{*}\right)$.

THEOREM 3.8. A $(1-\epsilon)$-MWM can be computed in time $O\left(m \epsilon^{-1} \log N\right)$.

Proof. Each Augmentation and Blossom Shrinking step takes $O(m)$ time using a modified depth-first search [Gabow and Tarjan 1991, Sect. 8]. (Finding a maximal set of augmenting paths is significantly simpler, conceptually, than finding a maximal set of minimum-length augmenting paths, as is done in Micali and Vazirani [1980] and Vazirani [1994].) Each Dual Adjustment step clearly takes linear time, as does the dual adjustment at the end of each scale. Scale $i<L=\log N$ begins with free vertices' $y$-values at $N / 2^{i+1}$ (or $N / 2-\delta_{0} / 2$, if $i=0$ ) and performs Dual Adjustments until they are $N / 2^{i+2}-\delta_{i} / 2$. Since $y$-values of free vertices are decremented by $\delta_{i} / 2$ in each Dual Adjustment step, there are exactly $\left(N / 2^{i+2}+\delta_{i} / 2\right) /\left(\delta_{i} / 2\right)=N /\left(2 \delta_{0}\right)+1=\epsilon^{\prime-1} / 2+1$ such steps. The final scale begins with free vertices' $y$-values at $N / 2^{L+1}$ and ends with them at zero, so there are fewer than $\left(N / 2^{L+1}\right) /\left(\delta_{L} / 2\right)=\epsilon^{\prime-1}$ Dual Adjustment steps. Lemma 3.7 guarantees that the final matching is a $(1-\epsilon)$-MWM for $\epsilon^{\prime} \leq \epsilon / 5$. Note that $M$ is not represented explicitly. After scale $L$, we have computed a nested set of blossoms $\Omega$ and a matching, call it $M^{\prime}$, in the contracted graph $G / \Omega$. Extending $M^{\prime}$ to the corresponding matching $M$ in $G$ is easily accomplished in $O(n)$ time by dissolving the blossoms in a top-down fashion. Hence, the total running time is $O\left(m \epsilon^{-1} \log N\right)$.

\subsection{A Linear-Time Algorithm}

Our $O\left(m \epsilon^{-1} \log N\right)$-time algorithm requires few modifications to run in linear time, independent of $N$. In fact, the algorithm as it appears in Figure 2 requires no modifications at all: we only need to change the definition of eligibility and, in each scale, refrain from scanning edges that cannot possibly be eligible. In light of Lemma 3.6(1), it is helpful to index edges according to the first scale in which they may be eligible.

Definition 3.9. Define $\mu_{i}=N / 2^{i+1}+\delta_{i}$, for $i<L$, and $\mu_{L}=0$. Define scale $(e)$ to be the $i$ such that $w(e) \in\left[\mu_{i}, \mu_{i-1}\right)$.

In other words, once we compute scale $(e)$ we can ignore $e$ in all scales $i<\operatorname{scale}(e)$, thereby saving time. The idea of our linear time algorithm is to forcibly ignore $e$ in 
scales $i>\operatorname{scale}(e)+\log \epsilon^{\prime-1}$. Ignoring an otherwise eligible edge can cause violations of near tightness and near dominance that increase with each dual adjustment. We shall prove that beyond scale $\operatorname{scale}(e)+\log \epsilon^{\prime-1}$, the magnitude of these violations is an $O\left(\epsilon^{\prime}\right)$ fraction of the weight of $e$, which is small enough to obtain a $\left(1-O\left(\epsilon^{\prime}\right)\right)$-MWM. Definition 3.10 redefines eligibility. The differences with Definition 3.2 are underlined.

Definition 3.10. Let $\gamma=\log \epsilon^{-1}$. At scale $i$, an edge $e$ is eligible if at least one of the following holds.

(1) $e \in E_{B}$ for some $B \in \Omega$.

(2) $e \notin M$ and $y z(e)=w_{i}(e)-\delta_{i}$ and scale $(e) \geq i-\gamma$.

(3) $e \in M$ and $y z(e)-w_{i}(e)$ is a nonnegative integer multiple of $\delta_{i}$ and scale $(e) \geq i-\gamma$.

Let $E_{\text {elig }}$ be the set of eligible edges and let $G_{\text {elig }}=\left(V, E_{\text {elig }}\right) / \Omega$ be the unweighted graph obtained by discarding ineligible edges and contracting root blossoms.

LEMMA 3.11. Consider an execution of the algorithm using Definition 3.10 of eligibility rather than Definition 3.2. Property 3.1(1,2,5) holds throughout the execution. For an edge $e$ and $k=$ scale(e), Property 3.1(3,4) (near domination and near tightness) hold in all scales $i \leq k+\gamma$ and thereafter in the following form. For each $e$, $y z(e)>\left(1-\epsilon^{\prime}\right) w_{i}(e)$ and for each matching or blossom edge $y z(e)<\left(1+6 \epsilon^{\prime}\right) w_{i}(e)$.

PROOF. The definition of eligibility has no bearing on Property $3.1(1,2,5)$ so they are maintained correctly. In scales $k$ through $k+\gamma$, Property $3.1(3,4)$ is maintained as the two definitions of eligibility are the same. At the beginning of scale $t=k+\gamma+1, e$ may still be eligible if it is in a blossom. The moment the blossom (if any) is dissolved, $e$ will become ineligible and remain so for the remainder of the computation. At the beginning of scale $t$, the $y$-values of free vertices are $N / 2^{t+1}$. From this moment on, the $y$-values of free vertices are incremented by a total of $\sum_{l \geq t+1} \delta_{l}$ (the dual adjustments following scales $t$ through $L-1$ ) and decremented a total of $N / 2^{t+1}+\sum_{l \geq t+1} \delta_{l}$ (in the Dual Adjustment steps following searches for augmenting paths and blossoms). We consider the effects of these adjustments on near tightness and near domination separately.

Near Tightness. Let $e=(u, v)$ be a type $j$ edge (matched or in a blossom) at the beginning of scale $t \leq L$. Each adjustment to $y$-values by some quantity $\Delta$ may cause $y z(e)$ to increase by $2 \Delta$. This clearly occurs in the dual adjustments following each scale as $y(u)$ and $y(v)$ are incremented by $\Delta$. Following a search for blossoms, it may be that $u, v \in \hat{V}_{i n}$, which would also cause $y(u)$ and $y(v)$ to each be incremented by $\Delta$. Putting this all together, it follows that at any scale $i \geq t=k+\gamma+1=k+\log \epsilon^{\prime-1}+1$,

$$
\begin{array}{rlr}
y z(e) & \leq w_{i}(e)+2\left(\delta_{j}-\delta_{t}\right)+2 \cdot\left(N / 2^{t+1}+2 \cdot \sum_{l \geq t+1} \delta_{l}\right) \\
& <w_{i}(e)+2\left(\delta_{k}-\delta_{t}\right)+2\left(\delta_{k+2}+2 \delta_{t}\right) & \\
& =w_{i}(e)+\left(2+1 / 2+\epsilon^{\prime}\right) \delta_{k} & j \geq k, \text { defn. of } t \\
& <w_{i}(e)+3 \delta_{k} & \text { defn. of } \delta_{t}=\epsilon^{\prime} \delta_{k} / 2 \\
& <\left(1+6 \epsilon^{\prime}\right) \cdot w_{i}(e) & w(e) \geq w_{i}(e)>N / 2^{k+1}=\left(2 \epsilon^{\prime}\right)^{-1} \cdot \delta_{k} .
\end{array}
$$

The inequality $y z(e)<\left(1+6 \epsilon^{\prime}\right) \cdot w_{i}(e)$ also holds if $i<t$ since, in this case, $y z(e) \leq$ $w_{i}(e)+2\left(\delta_{j}-\delta_{i}\right)<w_{i}(e)+2 \delta_{k}$. 
Near Domination. At the beginning of scale $t \leq L$, the $y$-values of free vertices are $N / 2^{t+1}$ and we have $y z(e) \geq w_{t}(e)-\delta_{t}$. In the remaining dual adjustments, $y z(e)$ will be incremented by $2\left(\sum_{l \geq t+1} \delta_{l}\right)$ (following each scale) and decremented by as much as $2\left(N / 2^{t+1}+\sum_{l \geq t+1} \delta_{l}\right)$. (This could occur if in each dual adjustment step, $u, v \in \hat{V}_{\text {out }}$.) Thus, at any scale $i \geq t=k+\gamma+1$,

$$
\begin{array}{rlr}
y z(e) & \geq w_{t}(e)-\delta_{t}-N / 2^{t} \\
& >w_{i}(e)-2 \delta_{t}-N / 2^{t} & \\
& =w_{i}(e)-\left(\epsilon^{\prime}+\frac{1}{2}\right) \delta_{k} & t=k+\gamma+1 ; \delta_{k}=\epsilon^{\prime} N / 2^{k} \\
& \geq w_{i}(e) \cdot\left(1-\left(\epsilon^{\prime}+\frac{1}{2}\right) /\left(\frac{1}{2 \epsilon^{\prime}}+1\right)\right) & w_{i}(e) \geq N / 2^{k+1}+\delta_{k}=\left(\frac{1}{2 \epsilon^{\prime}}+1\right) \delta_{k} \\
& =w_{i}(e) \cdot\left(1-\epsilon^{\prime}\right)
\end{array}
$$

If $t>L$, that is, Property 3.1(3) holds at the end of the computation, then $y z(e) \geq$ $w_{L}(e)-\delta_{L}=w(e)-\epsilon^{\prime} \geq\left(1-\epsilon^{\prime}\right) \cdot w(e)$.

THEOREM 3.12. A $(1-\epsilon)$-MWM can be computed in time $O\left(m \epsilon^{-1} \log \epsilon^{-1}\right)$.

PROOF. We execute the algorithm from Figure 2 where $G_{\text {elig }}$ refers to the eligible subgraph as defined in Definition 3.10. According to Lemmas 2.3 and 3.11, the algorithm returns a $\left(\left(1-\epsilon^{\prime}\right)\left(1+6 \epsilon^{\prime}\right)^{-1}\right)$-MWM, which, for $\epsilon^{\prime} \leq \epsilon / 7$, is a $(1-\epsilon)$-MWM. It remains to prove that the running time is $O\left(m \epsilon^{-1} \log \epsilon^{-1}\right)$.

Consider an edge $e$ with scale $(e)=k$. By Lemma 3.6(1) $e$ can be ignored in scales 0 through $k-1$. It can only appear in $G_{\text {elig }}$ (that is, as an eligible edge not in any blossom) in scales $k$ through $k+\gamma$. In scales $k+\gamma+1$ through $L=\log N, e$ may remain eligible, but only as a blossom edge. As soon as the blossoms containing $e$ are dissolved, $e$ can be ignored as it will never become eligible again. Since the algorithm need not spend any time examining edges in contracted blossoms, each edge actively participates in only $\gamma+1=\log \epsilon^{-1}+1$ scales, with $O\left(\epsilon^{-1}\right)$ iterations per scale. The total running time is therefore $O\left(m \epsilon^{-1} \log \epsilon^{-1}\right)$, provided that the scale $(\cdot)$ function can be efficiently computed.

Computing scale $(e)$ is tantamount to computing the most significant bit of $w(e)$. Once $\operatorname{MSB}(w(e))=\left\lfloor\log _{2} w(e)\right\rfloor$ is known, scale $(e)$ can be just one of two values. Recall from Section 2 that we can assume, without loss of generality, that $N \leq n^{2}$. Using $O(n)$ space and preprocessing time we can tabulate the MSB function on $\log n$-bit integers. We can then determine $\operatorname{MSB}(w(e))$ in $O(1)$ time with two table lookups.

\section{REFERENCES}

Alt, H., Blum, N., Mehlhorn, K., and Paul, M. 1991. Computing a maximum cardinality matching in a bipartite graph in time $O\left(n^{1.5} \sqrt{m / \log n}\right)$. Inf. Proc. Lett. 37, 4, 237-240.

Anderson, T., Owicki, S., Saxe, J., and Thacker, C. 1993. High speed switch scheduling for local area networks. ACM Trans. Comput. Syst. 11, 4, 319-352.

Andersson, A., Hagerup, T., Nilsson, S., and Raman, R. 1998. Sorting in linear time? J. Comput. Syst. Sci. 57, 1, 74-93.

Avis, D. 1978. Two greedy heuristics for the weighted matching problems. In Proceedings of the 9th Southeast Conference on Combinatorics, Graph Theory, and Computing (CongrNumer XXI). 65-76.

Avis, D. 1983. A survey of heuristics for the weighted matching problem. Networks 13, 475-493.

Balinski, M. L. 1969. Labelling to obtain a maximum matching. In Combinatorial Mathematics and Its Applications, R. C. Bose and T. A. Downing Eds., University of North Carolina Press, 585-602.

Bertsekas, D. P. 1981. A new algorithm for the assignment problem. Math. Prog. 21, 152-171.

Birkhoff, G. 1946. Tres observaciones sobre el elgebra lineal. Universidad Nacional de Tucuman, Revista A 5, 1-2, 147-151. 
Brogden, H. E. 1946. An approach to the problem of differential prediction. Psychometrika 11, 3, 139-154.

Brown, G. and von Neumann, J. 1950. Solutions of games by differential equations. In Contributions to the Theory of Games, H. Kuhn and A. Tucker Eds., Annals of Mathematical Studies Series, vol. 24. Princeton University Press, 73-79.

Cheriyan, J. and Mehlhorn, K. 1996. Algorithms for dense graphs and networks on the random access computer. Algorithmica 15, 6, 521-549.

Christofides, N. 1976. Worst case analysis of a new heuristic for the travelling salesman problem. Tech. rep., Graduate School of Industrial Administration, Carnegie Mellon University.

Cole, R., Hariharan, R., Lewenstein, M., and Porat, E. 2001. A faster implementation of the GoemansWilliamson clustering algorithm. In Proceedings of the 12th ACM-SIAM Symposium on Discrete Algorithms (SODA). 17-25.

Cunningham, W. H. and Marsh, III, A. B. 1978. A primal algorithm for optimum matching. Math. Prog. Study 8, 50-72.

Cygan, M., Gabow, H. N., and Sankowski, P. 2012. Algorithmic applications of Baur-Strassen's theorem: Shortest cycles, diameter and matchings. In Proceedings of the 53rd Annual IEEE Symposium on Foundations of Computer Science (FOCS). 531-540.

Dantzig, G. B. 1951. Application of the simplex method to the transportation problem. In Activity Analysis of Production and Allocation, Cowles Commission Monograph 13, T. C. Koopmans Ed., Wiley, New York, 359-373.

Desler, J. F. and Hakimi, S. L. 1969. A graph-theoretic approach to a class of integer-programming problems. Oper. Res. 17, 6, 1017-1033.

Dijkstra, E. W. 1959. A note on two problems in connexion with graphs. Numer. Math. 1, 269-271.

Dinic, E. A. 1970. Algorithm for solution of a problem of maximum flow in networks with power estimation. Soviet Math. Dokl. 11, 1277-1280.

Dinic, E. A. and Kronrod, M. A. 1969. An algorithm for the solution of the assignment problem. Soviet Math. Dokl. 10, 6, 1324-1326.

Drake, D. and Hougardy, S. 2003a. Improved linear time approximation algorithms for weighted matchings. In Proceedings of the 7th International Workshop on Randomization and Approximation Techniques in Computer Science (APPROX). Lecture Notes in Computer Science, vol. 2764, Springer. 14-23.

Drake, D. and Hougardy, S. 2003b. A simple approximation algorithm for the weighted matching problem. Inf. Proc. Lett. 85, 211-213.

Duan, R. and Pettie, S. 2010. Approximating maximum weight matching in near-linear time. In Proceedings of the 51st IEEE Symposium on Foundations of Computer Science (FOCS). 673-682.

Duan, R. and $\mathrm{Su}, \mathrm{H} .-\mathrm{H}$. 2012. A scaling algorithm for maximum weight matching in bipartite graphs. In Proceedings of the 23rd ACM-SIAM Symposium on Discrete Algorithms (SODA). 1413-1424.

Duff, I. S. and Gilbert, J. R. 2002. Maximum-weighted matching and block pivoting for symmetric indefinite systems. In Householder Symposium XV Book of Abstracts, 73-75.

Easterfield, T. E. 1946. A combinatorial algorithm. J. London Math. Soc. 21, 219-226. (Republished as: An algorithm for the allocation problem, Oper. Res. 11, 3, 123-129, 1960.)

Edmonds, J. 1965a. Maximum matching and a polyhedron with 0,1-vertices. J. Res. Nat. Bur. Stand. Sect. B 69B, 125-130.

Edmonds, J. 1965b. Paths, trees, and flowers. Canad. J. Math. 17, 449-467.

Edmonds, J. and Johnson, E. L. 1973. Matching, Euler tours, and the Chinese postman. Math. Prog. 5, 88-124.

Edmonds, J. and Karp, R. M. 1972. Theoretical improvements in algorithmic efficiency for network flow problems. J. ACM 19, 2, 248-264.

Feder, T. and Motwani, R. 1995. Clique partitions, graph compression and speeding-up algorithms. J. Comput. Syst. Sci. 51, 2, 261-272.

Ford, L. R. and Fulkerson, D. R. 1962. Flows in Networks. Princeton University Press.

Fredman, M. L. and Tarjan, R. E. 1987. Fibonacci heaps and their uses in improved network optimization algorithms. J. ACM 34, 3, 596-615.

Gabow, H. N. 1974. Implementation of algorithms for maximum matching on nonbipartite graphs. Ph.D. thesis, Stanford University.

Gabow, H. N. 1985a. A scaling algorithm for weighted matching on general graphs. In Proceedings of the 26th IEEE Symposium on Foundations of Computer Science (FOCS). 90-100.

Gabow, H. N. 1985b. Scaling algorithms for network problems. J. Comput. Syst. Sci. 31, 2, 148-168. 
Gabow, H. N. 1990. Data structures for weighted matching and nearest common ancestors with linking. In Proceedings of the 1st Annual ACM-SIAM Symposium on Discrete Algorithms (SODA). 434-443.

Gabow, H. N. and Pettie, S. 2002. The dynamic vertex minimum problem and its application to clusteringtype approximation algorithms. In Proceedings of the 8th Scandinavian Workshop on Algorithm Theory (SWAT). 190-199.

Gabow, H. N. and Tarjan, R. E. 1985. A linear-time algorithm for a special case of disjoint set union. J. Comput. Syst. Sci. 30, 2, 209-221.

Gabow, H. N. and Tarjan, R. E. 1989. Faster scaling algorithms for network problems. SIAM J. Comput. 18, $5,1013-1036$.

Gabow, H. N. and Tarjan, R. E. 1991. Faster scaling algorithms for general graph-matching problems. J. ACM 38, 4, 815-853.

Gabow, H. N., Galil, Z., and Spencer, T. H. 1989. Efficient implementation of graph algorithms using contraction. J. ACM 36, 3, 540-572.

Galil, Z., Micali, S., and Gabow, H. N. 1986. An $O(E V \log V)$ algorithm for finding a maximal weighted matching in general graphs. SIAM J. Comput. 15, 1, 120-130.

Giaccone, P., Leonardi, E., and Shah, D. 2005. On the maximal throughput of networks with finite buffers and its application to buffered crossbars. In Proceedings of the 24th INFOCOM. Vol. 2, 971-980.

Gleyzal, A. 1955. An algorithm for solving the transportation problem. J. Res. Nat. Bur. Standards 54, 4, $213-216$.

Goemans, M. X. and Williamson, D. P. 1995. A general approximation technique for constrained forest problems. SIAM J. Comput. 24, 2, 296-317.

Goldberg, A. V. and Karzanov, A. V. 2004. Maximum skew-symmetric flows and matchings. Math. Program., Ser. A 100, 537-568.

Goldberg, A. V. and Kennedy, R. 1997. Global price updates help. SIAM J. Disc. Math. 10, 4, 551-572.

Hadlock, F. 1975. Finding a maximum cut of a planar graph in polynomial time. SIAM J. Comput. 4, 3, 221-225.

Hagemann, M. and Schenk, O. 2006. Weighted matchings for preconditioning symmetric indefinite linear systems. SIAM J. Sci. Comput. 28, 2, 403-420.

Han, Y. 2002. Deterministic sorting in $O(n \log \log n)$ time and linear space. In Proceedings of the 34th ACM Symposium on Theory of Computing (STOC). 602-608.

Hanke, S. and Hougardy, S. 2010. New approximation algorithms for the weighted matching problem. Research rep. No. 101010, Research Institute for Discrete Mathematics, University of Bonn.

Harvey, N. 2009. Algebraic algorithms for matching and matroid problems. SIAM J. Comput. 39, 2, 679-702.

Hendrickson, B. and Leland, R. 1995. The Chaco user's guide: Version 2.0. Tech. rep. SAND94-2344, Sandia National Laboratories.

Hitchcock, F. L. 1941. The distribution of a product from several sources to numerous localities. J. Math. Phys. 20, 224-230.

Hoffman, A. J. and Markowitz, H. M. 1963. A note on shortest path, assignment, and transportation problems. Naval Res. Logistics Quart. 10, 1, 375-379.

Holtgrewe, M., Sanders, P., and Schulz, C. 2010. Engineering a scalable high quality graph partitioner. In Proceedings of the 24th IEEE Symposium on Parallel and Distributed Processing (IPDPS). 1-12.

Hopcroft, J. E. and Karp, R. M. 1973. An $n^{5 / 2}$ algorithm for maximum matchings in bipartite graphs. SIAM J. Comput. 2, 225-231.

Huang, C.-C. and Kavitha, T. 2012. Efficient algorithms for maximum weight matchings in general graphs with small edge weights. In Proceedings of the $23 \mathrm{rd}$ ACM-SIAM Symposium on Discrete Algorithms (SODA). 1400-1412.

Ibarra, O. H. and Moran, S. 1981. Deterministic and probabilistic algorithms for maximum bipartite matching via fast matrix multiplication. Inf. Proc. Lett. 13, 1, 12-15.

Iri, M., Murota, K., and Matsui, S. 1983. Heuristics for planar minimum-weight perfect matchings. Networks 13, 1, 67-92.

Johnson, D. B. 1975. Priority queues with update and finding minimum spanning trees. Inf. Proc. Lett. 4, 3, $53-57$.

Kantorovitch, L. 1942. On the translocation of masses. Doklady Akad. Nauk SSSR 37, 199-201.

Kao, M.-Y., Lam, T.-K., Sung, W.-K., and Ting, H.-F. 2001. A decomposition theorem for maximum weight bipartite matchings. SIAM J. Comput. 31, 1, 18-26.

Karypis, G. and Kumar, V. 1998. A fast and high quality multilevel scheme for partitioning irregular graphs. SIAM J. Sci. Comput. 20, 1, 359-392. 
Karzanov, A. V. 1973. An exact estimate of an algorithm for finding a maximum flow, applied to the problem "on representatives" [in Russian]. Probl. Cybernetics 5, 66-70. (English translation available at the author's website.)

Karzanov, A. V. 1976. Efficient implementations of Edmonds' algorithms for finding matchings with maximum cardinality and maximum weight. In Studies in Discrete Optimization, A. A. Fridman Ed., Nauka, Moscow, 306-327.

Kuhn, H. W. 1955a. The Hungarian method for the assignment problem. Naval Res. Log. Quart. 2, 83-97.

Kuhn, H. W. 1955b. On combinatorial properties of matrices. George Washington University Logistics Papers 11, 1-11. (English translation of J. Egerváry, Matrixok kombinatorius tulajdonságairól, Matematikai és Fizikai Lapok 38, 16-28, 1931.)

Kuhn, H. W. 1956. Variants of the Hungarian method for assignment problems. Naval Res. Log. Quart. 3, $253-258$.

Kuhn, H. W. and Baumol, W. J. 1962. An approximate algorithm for the fixed-charges transportation problem. Naval Res. Log. Quart. 9, 1-15.

Kurtzberg, J. M. 1962. On approximation methods for the assignment problem. J. ACM 9, 4, 419-439.

Kwan, M. K. 1962. Graphic programming using odd or even points. Chinese Math. 1, 273-277.

Lawler, E. 1976. Combinatorial Optimization: Networks and Matroids. Holt, Rinehart \& Winston, New York.

Leonardi, E., Mellia, M., Neri, F., and Marsan, M. A. 2003. Bounds on delays and queue lengths in inputqueued cell switches. J. ACM 50, 4, 520-550.

McKeown, N. 1999. The iSLIP scheduling algorithm for input-queued switches. IEEE/ACM Trans. Netw. 7, 2, 188-201.

McKeown, N., Anantharam, V., and Walrand, J. C. 1996. Achieving 100\% throughput in an input-queued switch. In Proceedings of the 15th INFOCOM. 296-302.

Micali, S. and Vazirani, V. V. 1980. An $O(\sqrt{|V|} \cdot|E|)$ algorithm for finding maximum matching in general graphs. In Proceedings of the 21st IEEE Symposium on Foundations of Computer Science (FOCS). 17-27.

Motzkin, T. S. 1956. The assignment problem. In Proceedings of the Symposia in Applied Mathematics VI, Numerical Analysis, 109-125.

Mucha, M. and Sankowski, P. 2004. Maximum matchings via Gaussian elimination. In Proceedings of the 45th IEEE Symposium on Foundations of Computer Science (FOCS). 248-255.

Munkres, J. 1957. Algorithms for the assignment and transportation problems. J. Soc. Indust. Appl. Math. 5, 32-38.

Ollivier, F. 2009. Looking for the order of a system of arbitrary ordinary differential equations. Appl. Algebra Eng. Commun. Comput. 20, 1, 7-32. (English translation of: C. G. J. Jacobi, De investigando ordine systematis aequationum differentialum vulgarium cujuscunque, Borchardt Journal für die reine und angewandte Mathematik 65, 4, pp. 297-320, 1865.)

Olschowka, M. and Neumaier, A. 1996. A new pivoting strategy for Gaussian elimination. Linear Algebra Appl. 240, 131-151.

Orlin, J. B. and Ahuja, R. K. 1992. New scaling algorithms for the assignment and minimum mean cycle problems. Math. Program. 54, 41-56.

Orlova, G. I. and Dorfman, Y. G. 1972. Finding the maximum cut in a planar graph. Eng. Cybernet. 10, 502-506.

Pellegrini, F. 2008. SCOTCH 5.1 user's guide. Technical report, LaBRI.

Pettie, S. 2005. Sensitivity analysis of minimum spanning trees in sub-inverse-Ackermann time. In Proceedings of the 16th International Symposium on Algorithms and Computation (ISAAC). 964-973.

Pettie, S. 2012. A simple reduction from maximum weight matching to maximum cardinality matching. Inf. Proc. Lett. 112, 23, 893-898.

Pettie, S. and Sanders, P. 2004. A simpler linear time $2 / 3-\epsilon$ approximation to maximum weight matching. Inf. Proc. Lett. 91, 6, 271-276.

Preis, R. 1999. Linear time 1/2-approximation algorithm for maximum weighted matching in general graphs. In Proceedings of the 16th Symposium on Theoretical Aspects of Computer Science (STACS). Lecture Notes in Computer Science, vol. 1563, 259-269.

Preis, R. and Diekmann, R. 1997. PARTY - A software library for graph partitioning. In Advances in Computational Mechanics with Parallel and Distributed Processing, B. H. V. Topping Ed., Civil-Comp Press, 63-71. 
Ramshaw, L. and Tarjan, R. E. 2012. A weight-scaling algorithm for min-cost imperfect matchings in bipartite graphs. In Proceedings of the 53rd Annual IEEE Symposium on Foundations of Computer Science, (FOCS). 581-590.

Reingold, E. M. and Supowit, K. J. 1983. Probabilistic analysis of divide-and-conquer heuristics for minimum weighted Euclidean matching. Networks 13, 49-66.

Reingold, E. M. and Tarjan, R. E. 1981. On a greedy heuristic for complete matching. SIAM J. Comput. 10, 4, 676-681.

Sanders, P. and Schulz, C. 2012. Distributed evolutionary graph partitioning. In Proceedings of the 14th Workshop on Algorithm Engineering and Experiments (ALENEX). 43-54.

Sankowski, P. 2009. Maximum weight bipartite matching in matrix multiplication time. Theoret. Comput. Sci. 410, 44, 4480-4488.

Schenk, O., Wächter, A., and Hagemann, M. 2007. Matching-based preprocessing algorithms to the solution of saddle-point problems in large-scale nonconvex interior-point optimization. Comput. Optim. Appl. 36, 2-3, 321-341.

Shah, D., Giaccone, P., and Prabhakar, B. 2002. Efficient randomized algorithms for input-queued switch scheduling. IEEE Micro 22, 1, 10-18.

Shah, D. and Kopikare, M. 2002. Delay bounds for the approximate maximum weight matching algorithm for input queued switches. In Proceedings of the 21st INFOCOM. 1024-1031.

Sharathkumar, R. and Agarwal, P. K. 2012. A near-linear time $\epsilon$-approximation algorithm for geometric bipartite matching. In Proceedings of the 44th Symposium on Theory of Computing Conference (STOC). 385-394.

Thorndike, R. L. 1950. The problem of classification of personnel. Psychometrika 15, 215-235.

Thorup, M. 1999. Undirected single-source shortest paths with positive integer weights in linear time. J. ACM 46, 3, 362-394.

Thorup, M. 2003. Integer priority queues with decrease key in constant time and the single source shortest paths problem. In Proceedings of the 35th ACM Symposium on Theory of Computing (STOC). 149-158.

Thorup, M. 2007. Equivalence between priority queues and sorting. J. ACM 54, 6.

Tomizawa, N. 1971. On some techniques useful for solution of transportation network problems. Networks 1, 2, 173-194.

Varadarajan, K. R. and Agarwal, P. K. 1999. Approximation algorithms for bipartite and non-bipartite matching in the plane. In Proceedings of the 10th ACM-SIAM Symposium on Discrete Algorithms (SODA). 805-814.

Vazirani, V. V. 1994. A theory of alternating paths and blossoms for proving correctness of the $O(\sqrt{V} E)$ general graph maximum matching algorithm. Combinatorica 14, 1, 71-109.

Vinkemeier, D. E. D. and Hougardy, S. 2005. A linear-time approximation algorithm for weighted matchings in graphs. ACM Trans. Algor. 1, 1, 107-122.

von Neumann, J. 1953. A certain zero-sum two-person game equivalent to the optimal assignment problem. In Contributions to the Theory of Games, H. W. Kuhn and A. W. Tucker Eds., Vol. II, Princeton University Press, 5-12.

Walshaw, C. and Cross, M. 2007. JOSTLE: Parallel multilevel graph-partitioning software - an overview. In Mesh Partitioning Techniques and Domain Decomposition Techniques, 27-58.

Williams, V. V. 2012. Multiplying matrices faster than Coppersmith-Winograd. In Proceedings of the 44th Symposium on Theory of Computing Conference (STOC). 887-898.

Witzgall, C. and Zahn, Jr., C. T. 1965. Modification of Edmonds' maximum matching algorithm. J. Res. Nat. Bur. Standards Sect. B 69B, 91-98.

Received December 2011; revised March 2013, September 2013; accepted September 2013 